\title{
Spacecraft Dynamics in the Vicinity of a Comet in a Rotating Frame
}

\author{
Sharyl M. Byram* \\ Department of Aerospace Engineering, University of Michigan, Ann Arbor, MI 48109 \\ and Daniel J. Scheeres ${ }^{\dagger}$ \\ Department of Aerospace Engineering Sciences, University of Colorado, Boulder, CO 80309
}

The focus of this paper is to identify and analyze stable Sun synchronous orbits in a Hill rotating frame which can be applied to any small body in the solar system. Outgassing jet accelerations will be considered for the case of comets. Once these orbits have been identified, control schemes to restrict their motion are explored.

\section{Introduction}

The recent rise in missions to small bodies has created a new set of dynamical problems to be solved. While many missions have flown by and investigated comets from a distance there has not been a mission to actually orbit and land on the surface. The need to safely orbit a comet has become important. NASA recently stressed the importance of obtaining a comet surface sample return. ${ }^{1}$ ESA's Rosetta mission is scheduled to deliver a lander to the surface of comet $67 \mathrm{P} /$ Churyumov-Gerasimenko in 2014. Both of these missions will involve a period of close proximity to the comet and potentially an orbital phase about the body. The focus of this work will be to identify and analyze stable Sun synchronous orbits in a rotating frame which can be used to orbit any small body in the solar system. Previous work has been done on this problem. Dankowicz ${ }^{2}$ has found these type of orbits in a non-rotating system and explored their stability previously. Scheeres and Marzari ${ }^{3}$ investigate the stability of such motions accounting for the orbit of the comet, while Scheeres ${ }^{4}$ investigated the definition and stability of sun-synchronous orbits started from the terminator plane. The current research combines the offset orbits of Dankowicz with the averaging analysis and rotation present in the Scheeres analysis. The stability of identified orbits will be tested for outgassing jet accelerations in the case of comets. Once these orbits have been shown to be stable, different control schemes to restrict their allowable motion will be explored.

\section{Equations of Motion}

Consider the case of a spacecraft in the vicinity of a comet and significantly far from any other celestial body. Assume that a negligible mass spacecraft is in the vicinity of a comet modelled as a point mass and subject to solar radiation pressure acting in the anti-sunward direction, $\hat{d}$. In an inertial frame, the equations of motion are:

$$
\ddot{\vec{r}}_{I}=\frac{\partial U}{\partial \vec{r}_{I}}+g \hat{d}
$$

where $\vec{r}_{I}$ is the position vector in an inertial frame, $U$ is the potential, and $g$ is the solar radiation pressure magnitude computed as: is computed as:

$$
g=\frac{\beta}{d^{2}}
$$

*PhD Candidate, Department of Aerospace Engineering, University of Michigan, Ann Arbor, MI 48109, byramsm@umich.edu.

${ }^{\dagger}$ A. Richard Seebass Chair, Professor, Department of Aerospace Engineering Sciences, University of Colorado, Boulder, CO 80309, scheeres@colorado.edu. 
where $\beta=(1+\eta) G 1 / B, G 1=110^{8} \mathrm{~kg}-\mathrm{km} 3 /\left(\mathrm{s}^{2}-\mathrm{m}^{2}\right), B$ is the spacecraft mass to area ratio in $\mathrm{kg} / \mathrm{m}^{2}, \eta$ is the reflectance of the spacecraft, and $d$ is the heliocentric distance of the comet in $k m .{ }^{4}$ The Hill equations of motion are appropriate given the dynamical setup. They are as follows in the orbit frame assuming a constant rotation about the Sun are (with $x$ along the Sun-comet line and $z$ out of the orbital plane):

$$
\begin{aligned}
\ddot{x} & =2 \omega \dot{y}+3 \omega^{2} x-\frac{\mu x}{r^{3}}+g \\
\ddot{y} & =-2 \omega \dot{x}-\frac{\mu y}{r^{3}} \\
\ddot{z} & =-\omega^{2} z-\frac{\mu z}{r^{3}}
\end{aligned}
$$

where $r=\sqrt{x^{2}+y^{2}+z^{2}}$. Future work will generalize this analysis to the case of an elliptic comet orbit about the sun. The angular momentum projected along the solar radiation direction, $\hat{x}$, is:

$$
h_{d}=\hat{x} \cdot(\vec{r} \times \dot{\vec{r}})
$$

where its projected time derivative has the form:

$$
\dot{h}_{d}=\hat{x} \cdot(\vec{r} \times \ddot{\vec{r}})
$$

If $\vec{r}=(x, y, z)$ and $\dot{\vec{r}}=(\dot{x}, \dot{y}, \dot{z})$, then this time derivative simply reduces to:

$$
\begin{gathered}
\dot{h_{d}}=\hat{x} \cdot\left(2 \omega \dot{x} z-\omega^{2} y z, g z+2 \omega \dot{y} z+4 \omega^{2} x z,-g y-2 \omega(x \dot{x}+y \dot{y})-3 \omega^{2} x y\right) \\
\dot{h_{d}}=2 \omega \dot{x} z-\omega^{2} y z
\end{gathered}
$$

A cylindrical frame will gain us more insight, therefore we apply the following transformation:

$$
\begin{aligned}
x & =x \\
y & =\rho \cos \theta \\
z & =\rho \sin \theta \\
\dot{x} & =\dot{x} \\
\dot{y} & =\dot{\rho} \cos \theta+\rho \dot{\theta} \sin \theta \\
\dot{z} & =\dot{\rho} \sin \theta-\rho \dot{\theta} \cos \theta
\end{aligned}
$$

Changing the variables to cylindrical coordinates results in:

$$
\dot{h_{d}}=2 \omega \dot{x} \rho \sin \theta-\frac{1}{2} \omega^{2} \rho^{2} \sin (2 \theta)
$$

Note that the average value of $\dot{h_{d}}$ over $2 \pi$ in $\theta$ is 0 due to the $\sin \theta$ and $\sin (2 \theta)$ terms if we assume constant values for $\rho$ and $\omega$ and a zero or periodic value for $\dot{x}$. This means that the projected angular momentum along $\hat{x}_{s}$ is conserved on average. This conservation of the projected angular momentum was found in Dankowicz ${ }^{2}$ for a non-rotating system implying that on average the dynamics in a rotating system have similarities to the non-rotating system.

Completely converting to the cylindrical coordinates, the equations of motion become:

$$
\begin{aligned}
\ddot{x} & =2 \omega(\dot{\rho} \cos \theta-\rho \dot{\theta} \sin \theta)+3 \omega^{2} x+g-\frac{x \mu}{r^{3}} \\
\ddot{\rho} & =-2 \dot{x} \omega \cos \theta+\rho \dot{\theta}^{2}-\rho \omega^{2} \sin ^{2} \theta-\frac{\rho \mu}{r^{3}} \\
\rho \ddot{\theta} & =2 \dot{x} \omega \sin \theta-2 \dot{\rho} \dot{\theta}-\rho \omega^{2} \sin \theta \cos \theta
\end{aligned}
$$

where $r=\sqrt{x^{2}+\rho^{2}}$. Note that a symmetry in the evolution of the $x$ and $\rho$ states exists for $+\dot{\theta}$ and $-\dot{\theta}$ when $\theta_{0}=0, \pi$. This is expected due to the term $\dot{\theta} \sin \theta=-\dot{\theta} \sin (-\theta)$ in the $\ddot{x}$ equation and $\dot{\theta}^{2}=(-\dot{\theta})^{2}$ in the $\ddot{\rho}$ equation. If these equations are averaged over $\theta$ as was done for the projected angular momentum, a

$$
2 \text { of } 19
$$


system similar to the non-rotating system case from Dankowicz ${ }^{2}$ is recovered with the additional terms of $3 \omega^{2} \bar{x}$ and $-\frac{1}{2} \bar{\rho} \omega^{2}$.

$$
\begin{aligned}
\ddot{\bar{x}} & =g-\frac{\bar{x} \mu}{r^{3}}+3 \omega^{2} \bar{x} \\
\ddot{\bar{\rho}} & =\bar{\rho}^{2}-\frac{1}{2} \bar{\rho} \omega^{2}-\frac{\bar{\rho} \mu}{r^{3}} \\
\ddot{\rho} \ddot{\bar{\theta}} & =-2 \dot{\bar{\rho}} \overline{\bar{\theta}}
\end{aligned}
$$

Note that equation 22 can be directly integrated as:

$$
\bar{\rho}^{2} \dot{\bar{\theta}}=h
$$

where $h$ is a constant and in this case is the averaged angular momentum magnitude. Therefore the averaged equations of motion reduce to functions of $\bar{x}$ and $\bar{\rho}$ with constant $h$ :

$$
\begin{aligned}
& \ddot{\bar{x}}=g-\frac{\bar{x} \mu}{\bar{r}^{3}}+3 \omega^{2} \bar{x} \\
& \ddot{\bar{\rho}}=\bar{\rho}\left(\frac{h^{2}}{\bar{\rho}^{4}}-\frac{1}{2} \omega^{2}-\frac{\mu}{\bar{r}^{3}}\right)
\end{aligned}
$$

To find the equilibrium solutions to this set of equations, we set $\ddot{\bar{x}}$ and $\ddot{\bar{\rho}}$ to zero and find that the following conditions from algebraic manipulation of the equations of motion must hold:

$$
\begin{aligned}
3 \omega^{2} & =\frac{\mu}{\bar{r}^{3}}-\frac{g}{\bar{x}} \\
\frac{7}{2} \omega^{2} & =\frac{h}{\bar{\rho}^{4}}-\frac{g}{\bar{x}}
\end{aligned}
$$

For a given value of $\bar{x}_{0}$, then the variables $\bar{\rho}_{0}$ and $\dot{\bar{\theta}}_{0}$ can be found as functions of $\bar{x}_{0}$ yielding a family of Sun synchronous circular orbits perpendicular to the Sun line:

$$
\begin{aligned}
\bar{\rho}_{0} & =\sqrt{\left(\frac{\bar{x}_{0} \mu}{3 \omega^{2} \bar{x}_{0}+g}\right)^{2 / 3}-\bar{x}_{0}^{2}} \\
\dot{\bar{\theta}}_{0} & =\frac{7 \omega^{2}}{2}+\frac{g}{\bar{x}_{0}}
\end{aligned}
$$

Note that these solutions are dependent on the values of $\mu$ and $g$ and will result in different family profiles for each system. It may be possible for these solutions not to exist for any value of $\bar{x}_{0}$ depending on the values of $\mu$ and $g$ for a given system. An example profile of the family of circular orbits can be seen in figure 1 while figures 2- 5 illustrate the trajectory examples in the vicinity of these circular orbits. Note that the larger the $x$ offset, the more it deviates from the averaged solution. These orbits are generalizations of the terminator orbits described in Scheeres. ${ }^{4}$

\section{Zero Velocity Curves}

To gain insight into the dynamics of the system, it is instructive to construct the zero velocity curves that may exist. The Hill equations of motion in cylindrical coordinates have a Jacobi integral of:

$$
J=\frac{1}{2}\left(\dot{x}^{2}+\dot{\rho}^{2}+\rho^{2} \dot{\theta}^{2}\right)-\frac{\mu}{r}-\frac{1}{2} \omega^{2}\left(3 x^{2}-\rho^{2} \sin (\theta)^{2}\right)-g x
$$

Averaging this over $\theta$ as before yields:

$$
\bar{J}=\frac{1}{2}\left(\dot{\bar{x}}^{2}+\dot{\bar{\rho}}^{2}\right)-\frac{\mu}{\bar{r}}-\frac{1}{2} \omega^{2}\left(3 \bar{x}^{2}-\frac{1}{2} \bar{\rho}^{2}\right)-g \bar{x}+\frac{h^{2}}{2 \bar{\rho}^{2}}
$$

$$
3 \text { of } 19
$$



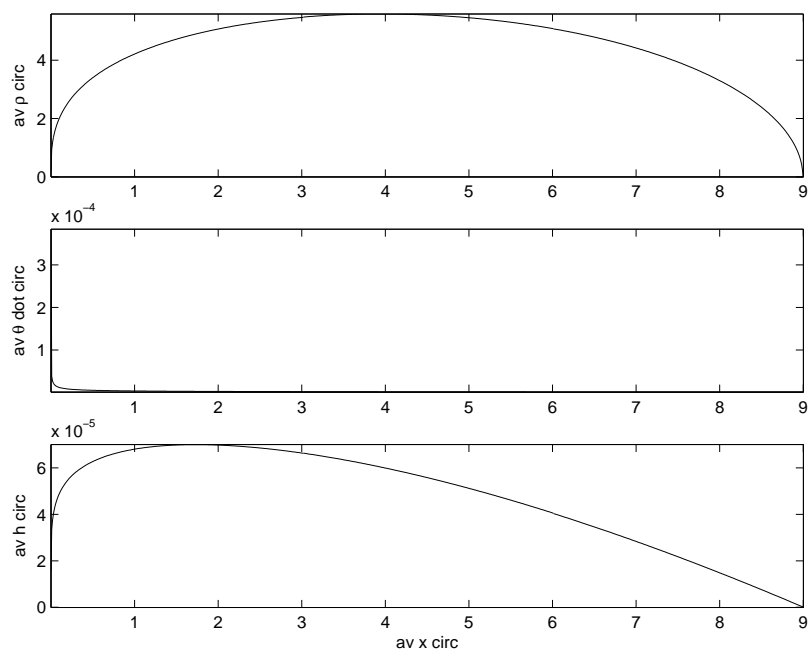

Figure 1. Example of circular orbit equilibrium solutions of the averaged equations as a function of the $x$ offset.

where $\dot{\bar{\theta}}=h / \bar{\rho}^{2}$. Note this is same Jacobi integral can be obtained by examining the averaged equations alone where $V=\frac{\mu}{\bar{r}}+\frac{1}{2} \omega^{2}\left(3 \bar{x}^{2}-\frac{1}{2} \bar{\rho}^{2}\right)+g \bar{x}-\frac{h^{2}}{2 \bar{\rho}^{2}}$. To find the zero velocity curves, we set the Jacobi integral to some arbitrary constant, $C$, and the velocities to zero.

$$
C=-\frac{\mu}{\bar{r}}-\frac{1}{2} \omega^{2}\left(3 \bar{x}^{2}-\frac{1}{2} \bar{\rho}^{2}\right)-g \bar{x}+\frac{h^{2}}{2 \bar{\rho}^{2}}
$$

Note that $C=-V$ as expected and therefore for a given $C$, the spacecraft's motion can occur where

$$
C+V \geq 0
$$

To find the zero velocity curves, we consider the equation:

$$
C_{m}+\frac{\mu}{\bar{r}}+\frac{1}{4} \omega^{2} \bar{\rho}^{2}-\frac{h^{2}}{2 \bar{\rho}^{2}}=0
$$

where

$$
C_{m}=C+\frac{3}{2} \omega^{2} \bar{x}^{2}+g \bar{x}
$$

$C_{m}$ is considered a constant for a given $\bar{x}$. Rearranging equation 35 , the zero velocity curves can be found by solving for the roots of the following polynomial.

$$
\begin{aligned}
0= & \left(\frac{1}{16} \omega^{4}\right) \bar{\Gamma}^{5}+\left(-\frac{1}{2} C_{m} \omega^{2}+\frac{1}{16} \omega^{4} \bar{x}^{2}\right) \bar{\Gamma}^{4}+\left(C_{m}^{2}+\frac{1}{4} h^{2} \omega^{2}-\frac{1}{2} C_{m} \omega^{2} \bar{x}^{2}\right) \bar{\Gamma}^{3} \\
& +\left(-\mu^{2}-C_{m} h^{2}+C_{m}^{2} \bar{x}^{2}+\frac{1}{4} h^{2} \omega^{2} \bar{x}^{2}\right) \bar{\Gamma}^{2}+\left(\frac{1}{4} h^{4}-C_{m} h^{2} \bar{x}^{2}\right) \bar{\Gamma}+\left(\frac{1}{4} h^{4} \bar{x}^{2}\right)
\end{aligned}
$$

Note that only $\bar{\rho} \geq 0$ is appropriate for cylindrical coordinates so the variable $\bar{\Gamma}=\bar{\rho}^{2}$ is used. For a given, $\bar{x}, h, C$, and $g$, the zero velocity curves can be computed. Figure $[6$ (figure 7 for the detail) shows the detail of the zero velocity curves for various circular orbit equilibrium solutions within a family. Note there exists a limit to where there is allowable motion to the Sun side of the equilibrium solution before the equilibrium solution becomes a stay alone point of the zero velocity curves. This limit appears to be a stable/unstable boundary for the circular orbit equilibrium solutions although this will be analytically verified in the next section. Figure 8 shows the curves for differing values of $C$ while holding $h, \omega$, and $g$ constant. For an $h$ value associated with a stable equilibrium solution, increasing the energy opens up the allowable area for motion around the equilibrium point and eventually leads to the two areas merging together at conjugate unstable equilibrium point which has the same $h$ value.

\section{4 of 19}



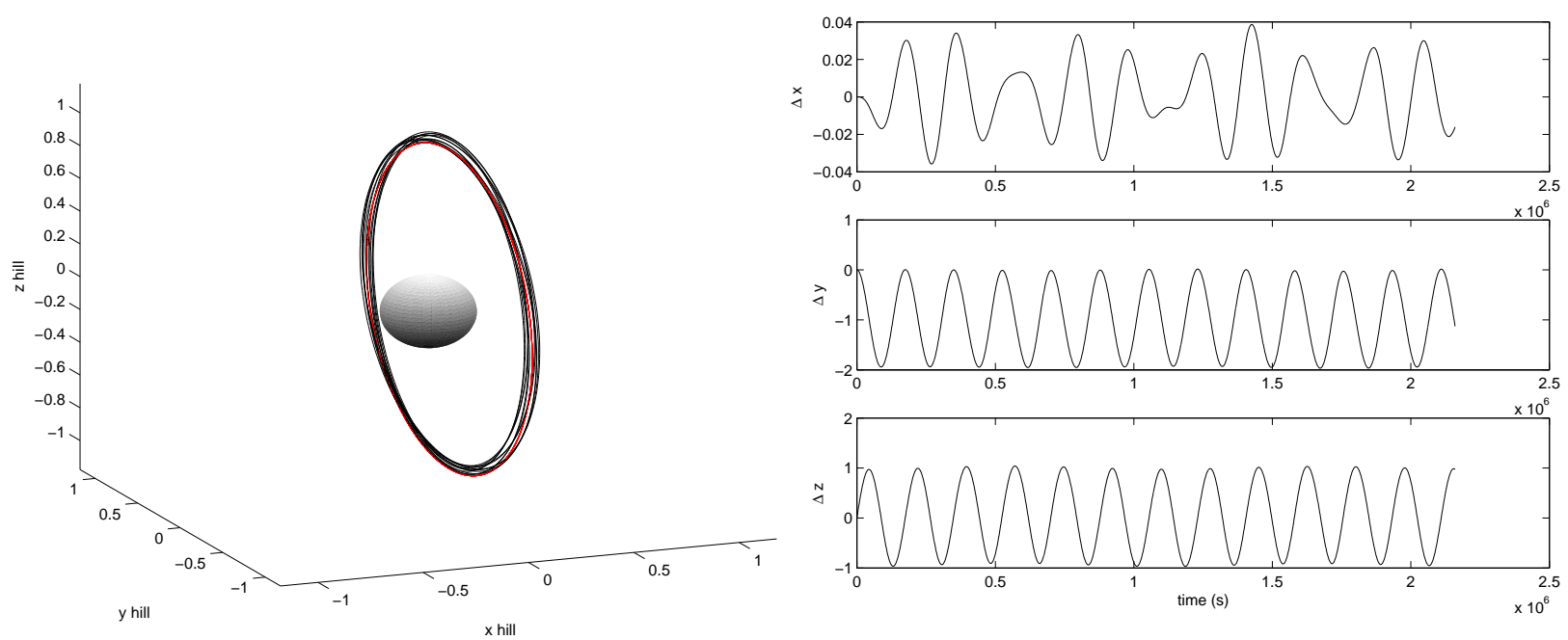

Figure 2. Initial offset $\left(x_{0}=.1 \mathrm{~km}\right)$ orbit about a comet with a regular gravity field in the rotating frame. Black = full Hill equations of motion trajectory. Red = averaged Hill equations of motion trajectory. Shown with full equations' component deviation $(\Delta x, \Delta y, \Delta z)$ from the averaged solution.
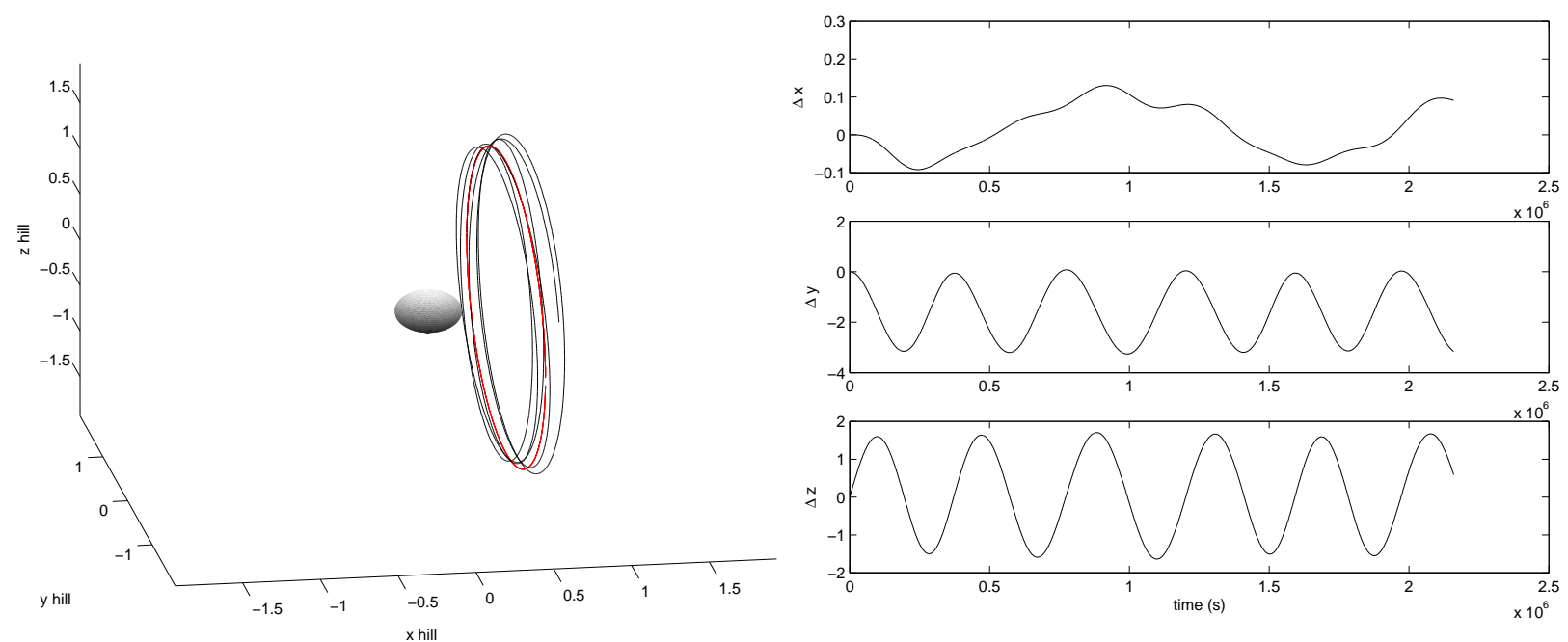

Figure 3. Initial offset $\left(x_{0}=.5 \mathrm{~km}\right)$ orbit about a comet with a regular gravity field in the rotating frame. Black $=$ full Hill equations of motion trajectory. Red $=$ averaged Hill equations of motion trajectory. Shown with full equations' component deviation $(\Delta x, \Delta y, \Delta z)$ from the averaged solution. 

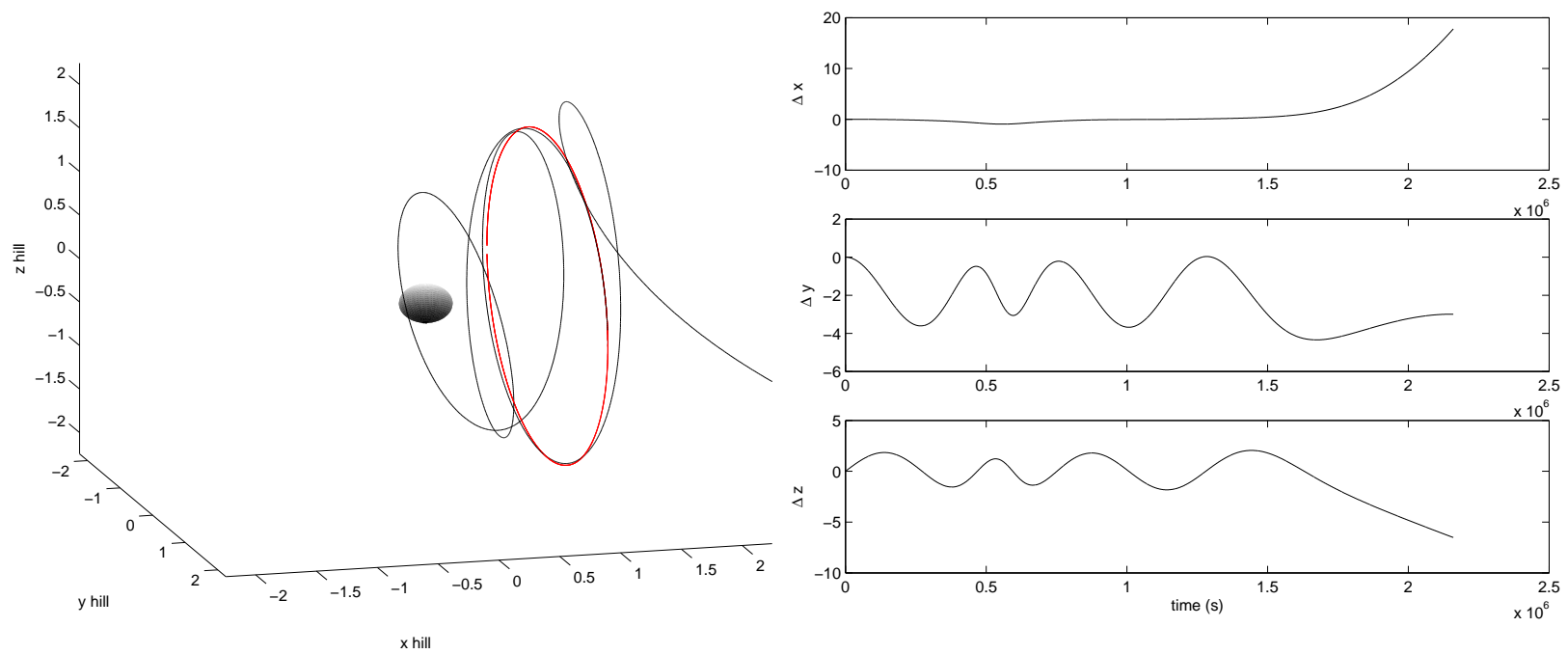

Figure 4. Initial offset $\left(x_{0}=1 \mathrm{~km}\right)$ orbit about a comet with a regular gravity field in the rotating frame. Black $=$ full Hill equations of motion trajectory. Red = averaged Hill equations of motion trajectory. Shown with full equations' component deviation $(\Delta x, \Delta y, \Delta z)$ from the averaged solution.
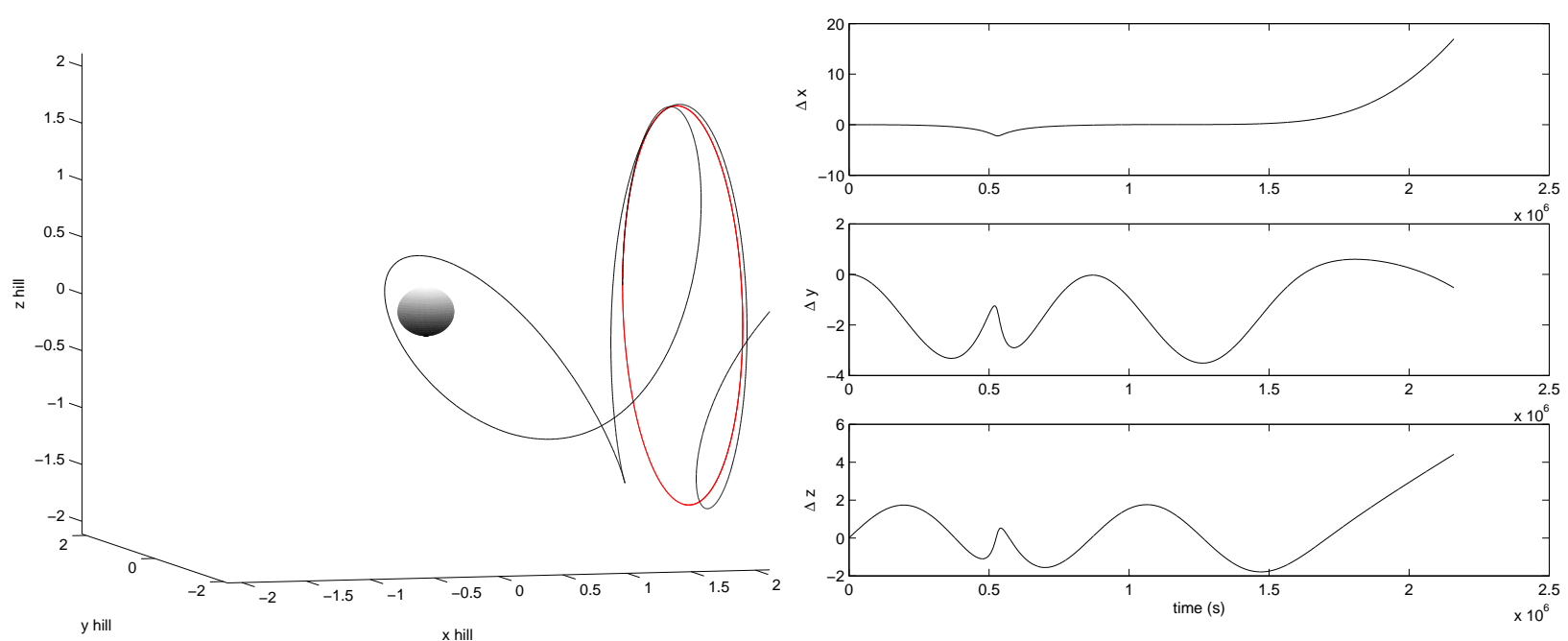

Figure 5. Initial offset $\left(x_{0}=2 \mathrm{~km}\right)$ orbit about a comet with a regular gravity field in the rotating frame. Black $=$ full Hill equations of motion trajectory. Red $=$ averaged Hill equations of motion trajectory. Shown with full equations' component deviation $(\Delta x, \Delta y, \Delta z)$ from the averaged solution. 


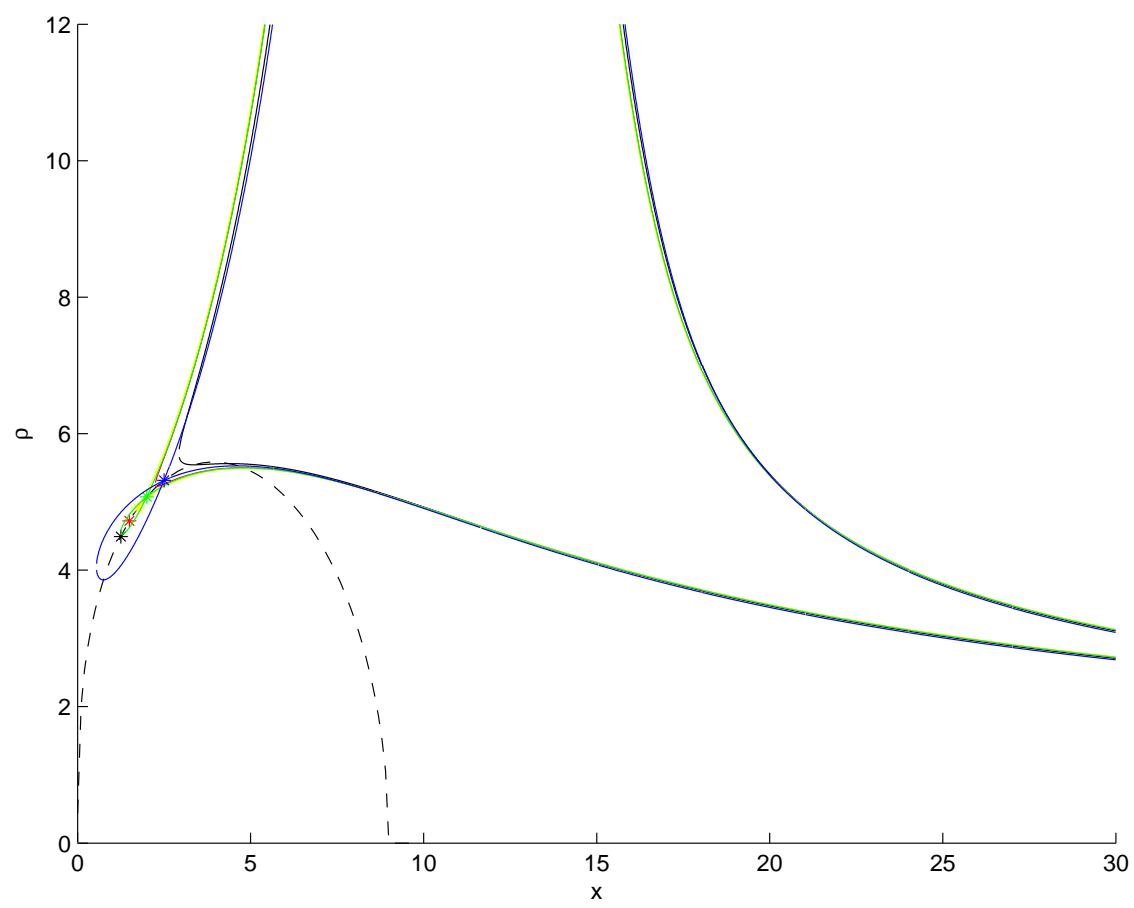

Figure 6. Zero velocity curves for various equilibrium solutions. ${ }^{*}=$ equilibrium solution at each $C$ energy level. Dashed $=$ circular orbit equilibrium solutions curve. Black $\rightarrow$ Blue $=$ decreasing $C$ value.

The shape or curvature of the zero velocity curves is determined by the solar radiation pressure magnitude, $g$. Larger values of $g$ will tend to straighten the upper portion of the zero velocity curve and shrink the area of allowable motion for a fixed value of $h$ as seen in figure 9 for a system with constant $h$ and $\omega$ and varied the solar radiation pressure magnitude.

The zero velocity curves appear to have an open shape, so it is only natural to determine if there exists any other conditions for the curves to close up and bound the motion of the spacecraft inside. First, consider the partial derivatives of the Jacobi integral:

$$
\begin{gathered}
\bar{J}_{\bar{x}}=\frac{\partial \bar{J}}{\partial \bar{x}}=-g-3 \omega^{2} \bar{x}+\frac{\mu \bar{x}}{\left(\bar{\rho}^{2}+\bar{x}^{2}\right)^{3 / 2}} \\
\bar{J}_{\bar{\rho}}=\frac{\partial \bar{J}}{\partial \bar{\rho}}=-\frac{h^{2}}{\bar{\rho}^{3}}+\frac{1}{2} \omega^{2} \bar{\rho}+\frac{\mu \bar{\rho}}{\left(\bar{\rho}^{2}+\bar{x}^{2}\right)^{3 / 2}}
\end{gathered}
$$

To find a point of closure, $\bar{J}_{\bar{x}}=0$ and $\bar{J}_{\bar{\rho}}=0$ must hold. Note that equations 38 and 39 are exactly the conditions used to find the circular orbit equilibrium solutions. First consider the case of negligible solar radiation pressure, $g=0$, these partial equations become:

$$
\begin{gathered}
-3 \omega^{2} \bar{x}+\frac{\mu \bar{x}}{\left(\bar{\rho}^{2}+\bar{x}^{2}\right)^{3 / 2}}=0 \\
-\frac{h^{2}}{\bar{\rho}^{3}}+\frac{1}{2} \omega^{2} \bar{\rho}+\frac{\mu \bar{\rho}}{\left(\bar{\rho}^{2}+\bar{x}^{2}\right)^{3 / 2}}=0
\end{gathered}
$$

Rearranging yields:

$$
\begin{gathered}
3 \omega^{2}=\frac{\mu}{\left(\bar{\rho}^{2}+\bar{x}^{2}\right)^{3 / 2}} \\
\frac{h^{2}}{\bar{\rho}^{4}}-\frac{1}{2} \omega^{2}=\frac{\mu}{\left(\bar{\rho}^{2}+\bar{x}^{2}\right)^{3 / 2}}
\end{gathered}
$$

$$
7 \text { of } 19
$$




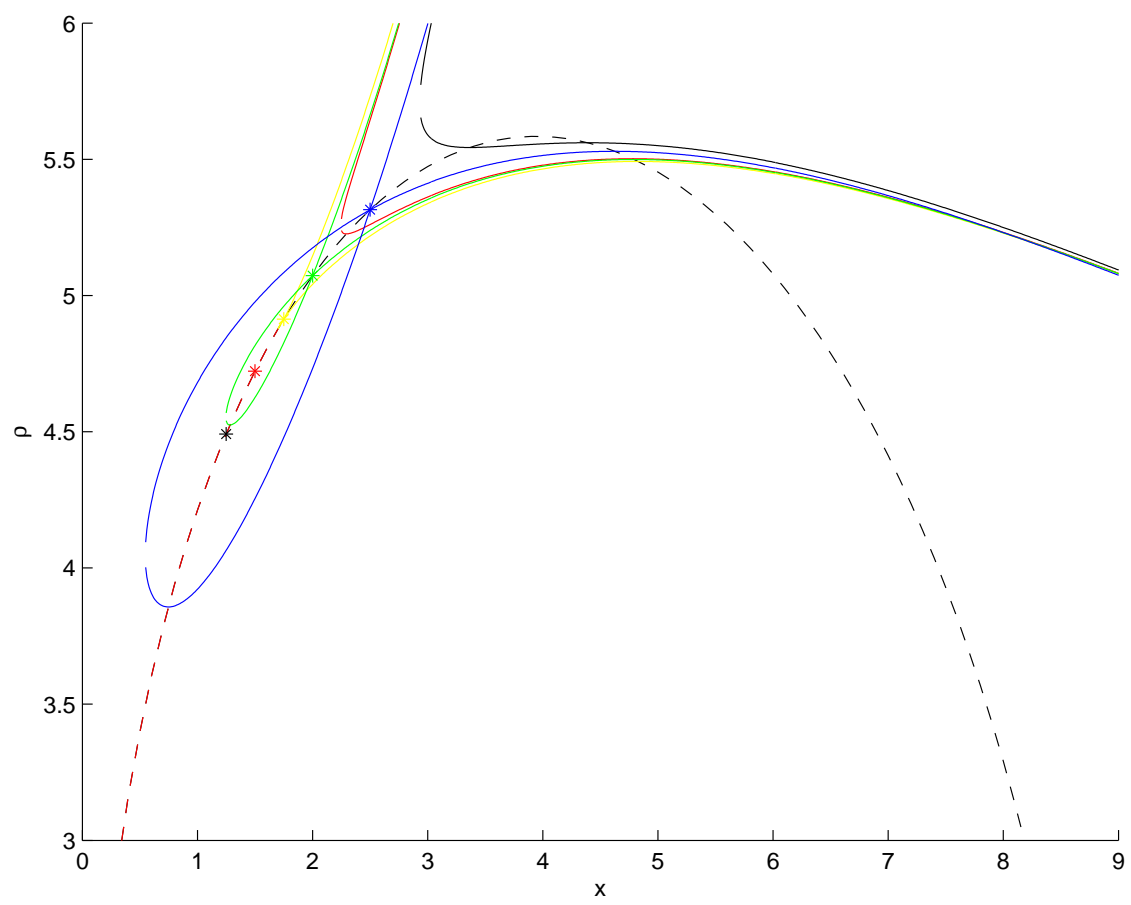

Figure 7. Zero velocity curves detail for various equilibrium solutions. ${ }^{*}=$ equilibrium solution at each $C$ energy level. Dashed $=$ circular orbit equilibrium solutions curve. Black $\rightarrow$ Blue $=$ decreasing $C$ value.

By substitution, the following solution is found:

$$
\begin{gathered}
\bar{\rho}^{4}=\frac{2 h^{2}}{7 \omega^{2}} \\
\bar{x}^{2}=\left(\frac{\mu}{3 \omega^{2}}\right)^{2 / 3}-\sqrt{\frac{2}{7}} \frac{h}{\omega}
\end{gathered}
$$

Note that for a real valued solution to exist, $\bar{x}^{2}>0$. This condition on $\bar{x}$ becomes a condition on the angular momentum magnitude, $h$, for the system:

$$
h<\left(\frac{\mu}{3 \omega^{2}}\right)^{2 / 3} \sqrt{\frac{7}{2}} \omega
$$

Since we are mostly interested in the circular orbit equilibrium solutions, we can substitute $h=\bar{\rho}_{0}^{2} \dot{\bar{\theta}}_{0}$, using the solutions for $\bar{\rho}_{0}$ and $\dot{\bar{\theta}}_{0}$ into this condition and find the condition to be a function of $\bar{x}_{0}$ :

$$
\left(\left(\frac{\bar{x}_{0} \mu}{3 \omega^{2} \bar{x}_{0}+g}\right)^{2 / 3}-\bar{x}_{0}^{2}\right)\left(\frac{7 \omega^{2}}{2}+\frac{g}{\bar{x}_{0}}\right)<\left(\frac{\mu}{3 \omega^{2}}\right)^{2 / 3} \sqrt{\frac{7}{2}} \omega
$$

If we now consider that the solar radiation pressure is non-negligible then the rearranged equations are:

$$
\begin{gathered}
3 \omega^{2}+\frac{g}{\bar{x}}=\frac{\mu}{\left(\bar{\rho}^{2}+\bar{x}^{2}\right)^{3 / 2}} \\
\frac{h^{2}}{\bar{\rho}^{4}}-\frac{1}{2} \omega^{2}=\frac{\mu}{\left(\bar{\rho}^{2}+\bar{x}^{2}\right)^{3 / 2}}
\end{gathered}
$$

These equations are the same as the conditions for the circular orbit equilibrium solutions. This implies that closure points occur at equilibrium solutions with the same angular momentum magnitude as predicted by the zero velocity curves and that there does not exist any other closure points of the system allowing a spacecraft to escape.

$$
8 \text { of } 19
$$




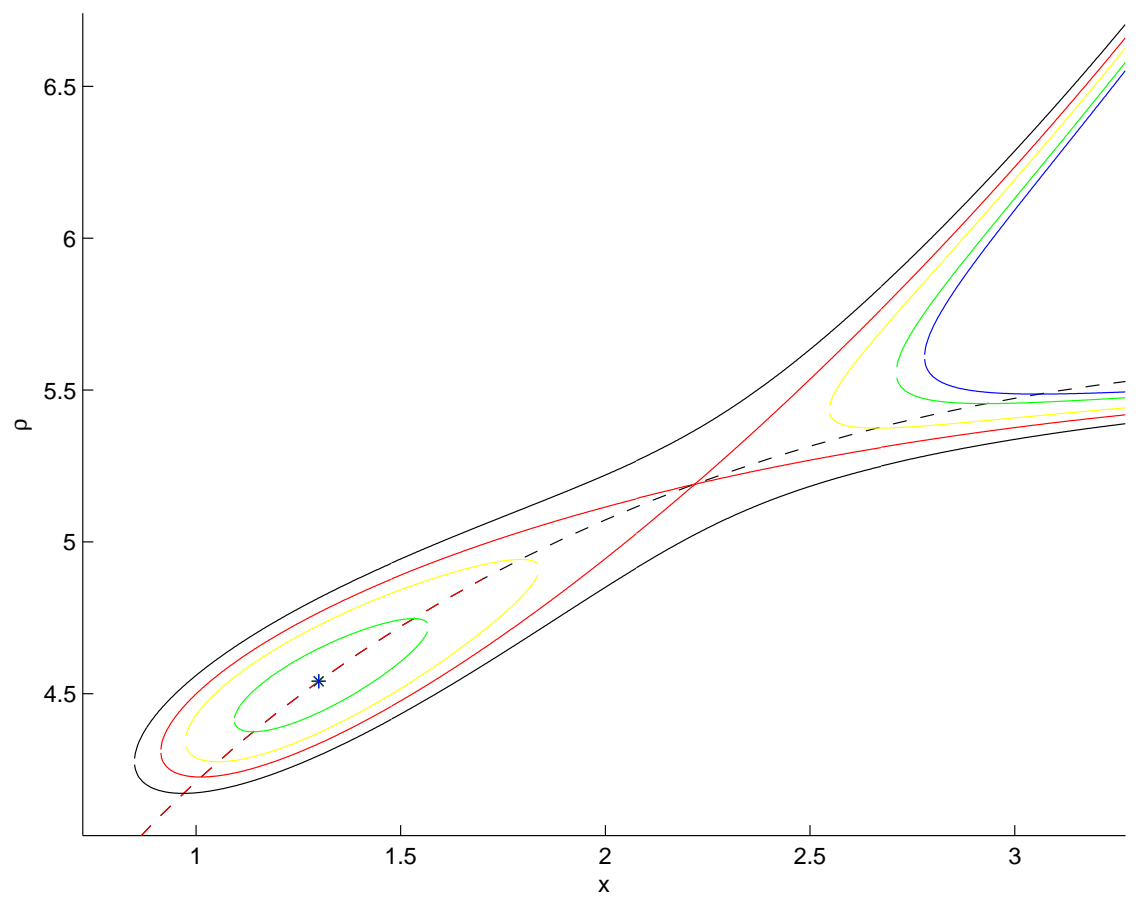

Figure 8. Zero velocity curves with constant $h .{ }^{*}=$ equilibrium solution at each $C$ energy level. Dashed $=$ circular orbit equilibrium solutions curve $(\operatorname{Red}=$ stable, Black $=$ unstable $)$. Black $\rightarrow$ Blue $=$ decreasing $C$ value.

\section{Stability of Averaged Equations of Motion}

Since the zero velocity curves have provided a graphical look that the stability of the system, let's now determine it analytically. To begin the averaged equations are linearized about the circular orbit $\left(\bar{\rho}_{0}, \bar{x}_{0}\right.$, and $\dot{\bar{\theta}}_{0}$ from the above relations) yielding the following linear equations with time invariant coefficients:

$$
\begin{aligned}
\delta \ddot{\bar{x}} & =\left(\frac{3 \bar{x}_{0}^{2} \mu}{\bar{r}_{0}^{5}}-\frac{\mu}{\bar{r}_{0}^{3}}+3 \omega^{2}\right) \delta \bar{x}+\left(\frac{3 \bar{x}_{0} \bar{\rho}_{0} \mu}{\bar{r}_{0}^{5}}\right) \delta \bar{\rho} \\
\delta \ddot{\bar{\rho}} & =\left(\frac{3 \bar{x}_{0} \bar{\rho}_{0} \mu}{\bar{r}_{0}^{5}}\right) \delta \bar{x}+\left(\frac{3 \bar{\rho}_{0}^{2} \mu}{\bar{r}_{0}^{5}}-\frac{\mu}{\bar{r}_{0}^{3}}+\dot{\bar{\theta}}_{0}^{2}-\frac{1}{2} \omega^{2}\right) \delta \bar{\rho}+\left(2 \bar{\rho}_{0} \dot{\bar{\theta}}_{0}\right) \delta \dot{\bar{\theta}} \\
\delta \ddot{\bar{\theta}} & =\left(\frac{2 \dot{\bar{\rho}}_{0} \dot{\bar{\theta}}_{0}}{\bar{\rho}_{0}^{2}}\right) \delta \bar{\rho}+\left(\frac{-2 \dot{\bar{\theta}}_{0}}{\bar{\rho}_{0}}\right) \delta \dot{\bar{\rho}}+\left(\frac{-2 \dot{\bar{\rho}}_{0}}{\bar{\rho}_{0}}\right) \delta \dot{\bar{\theta}}
\end{aligned}
$$

Note that for a circular orbit, $\dot{\bar{\rho}}_{0}=0$ therefore equation 52 becomes:

$$
\delta \ddot{\bar{\theta}}=\left(\frac{-2 \dot{\bar{\theta}}_{0}}{\bar{\rho}_{0}^{2}}\right) \delta \dot{\bar{\rho}}
$$

For notational simplicity, these equations are rewritten as:

$$
\begin{aligned}
\delta \ddot{\bar{x}} & =a \delta \bar{x}+b \delta \bar{\rho} \\
\delta \ddot{\bar{\rho}} & =c \delta \bar{x}+d \delta \bar{\rho}+e \delta \dot{\bar{\theta}} \\
\delta \ddot{\bar{\theta}} & =f \delta \dot{\bar{\rho}}
\end{aligned}
$$

$$
9 \text { of } 19
$$




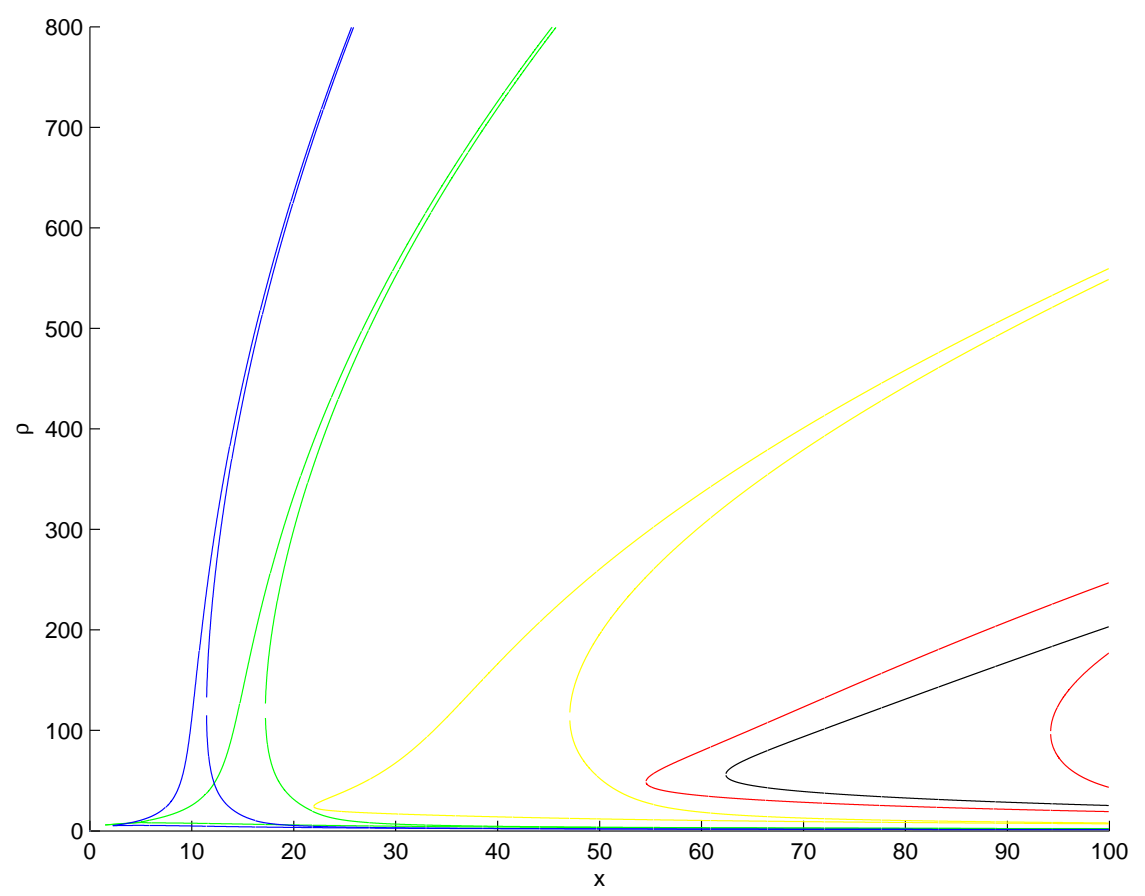

Figure 9. Zero Velocity Curves for Fixed $\omega$ varied $g$. Black $\rightarrow$ Blue $=$ Increasing $g$ Value.

where $a, b, c, d, e, f$ are defined by the original linearized averaged equations and $c=b$. In state space form, the linearized averaged equations are:

$$
\begin{gathered}
\left(\begin{array}{c}
\delta \dot{\bar{x}} \\
\delta \dot{\bar{\rho}} \\
\dot{\bar{\theta}} \\
\delta \ddot{\bar{x}} \\
\delta \ddot{\bar{\rho}} \\
\delta \ddot{\bar{\theta}}
\end{array}\right)=\left(\begin{array}{llllll}
0 & 0 & 0 & 1 & 0 & 0 \\
0 & 0 & 0 & 0 & 1 & 0 \\
0 & 0 & 0 & 0 & 0 & 1 \\
a & b & 0 & 0 & 0 & 0 \\
b & d & 0 & 0 & 0 & e \\
0 & 0 & 0 & 0 & f & 0
\end{array}\right)\left(\begin{array}{c}
\delta \bar{x} \\
\delta \bar{\rho} \\
\delta \bar{\theta} \\
\delta \overline{\bar{x}} \\
\delta \dot{\bar{\rho}} \\
\delta \dot{\bar{\theta}}
\end{array}\right) \\
\overline{\mathbf{X}}=\mathbf{A} \overline{\mathbf{X}}
\end{gathered}
$$

To determine the stability of this system, let's examine the eigenvalues of the A matrix, which are determined by its characteristic polynomial:

$$
\lambda^{6}+(-a-d-e f) \lambda^{4}+\left(-b^{2}+a d+a e f\right) \lambda^{2}=0
$$

It is obvious from the characteristic polynomial that there are two eigenvalues at 0 . Therefore, the system is by definition unstable, but the eigenvector associated with the zero eigenvalues is $(0,0,1,0,0,0)$ or the $\theta$ direction which does not realistically effect the stability of the circular orbit. Therefore, it is the other eigenvalues which will be used to determine the stability of the orbit. Eliminating the zero roots results in:

$$
\lambda^{4}+(-a-d-e f) \lambda^{2}+\left(-b^{2}+a d+a e f\right)=0
$$

which has the roots:

$$
\lambda= \pm \sqrt{\frac{(a+d+e f) \pm \sqrt{\left(4 b^{2}+(-a+d+e f)^{2}\right)}}{2}}
$$

where

$$
\left(4 b^{2}+(-a+d+e f)^{2}\right)=\frac{36 \mu^{2} \bar{x}_{0}^{2} \bar{\rho}_{0}^{2}}{\left(\bar{x}_{0}^{2}+\bar{\rho}_{0}^{2}\right)^{5}}+\left(3 \dot{\bar{\theta}}_{0}^{2}+\frac{3 \mu\left(\bar{x}_{0}-\bar{\rho}_{0}\right)\left(\bar{x}_{0}+\bar{\rho}_{0}\right)}{\left(\bar{x}_{0}^{2}+\bar{\rho}_{0}^{2}\right)^{5 / 2}}+\frac{7 \omega^{2}}{2}\right)^{2}>0
$$

$$
10 \text { of } 19
$$




$$
(a+d+e f)=-3 \dot{\bar{\theta}}_{0}^{2}+\frac{\mu}{\left(\bar{x}_{0}^{2}+\bar{\rho}_{0}^{2}\right)^{3 / 2}}+\frac{5 \omega^{2}}{2}
$$

With two of these roots having positive signs, only a purely imaginary roots will allow for a stable solution and they depend on the comet's specific orbit. Therefore to have purely imaginary roots, the following condition needs to hold for the comet's orbit:

$$
(a+d+e f)+\sqrt{4 b^{2}+(-a+d+e f)^{2}}<0
$$

or

$$
(a+d+e f)<-\sqrt{4 b^{2}+(-a+d+e f)^{2}}
$$

since $\sqrt{4 b^{2}+(-a+d+e f)^{2}}>0$ always.

As an example, figure 10 illustrates the stable and unstable circular orbit equilibrium solutions based on this analysis. The eigenvalues are indeed either zero or purely imaginary for the stable solutions in this example.

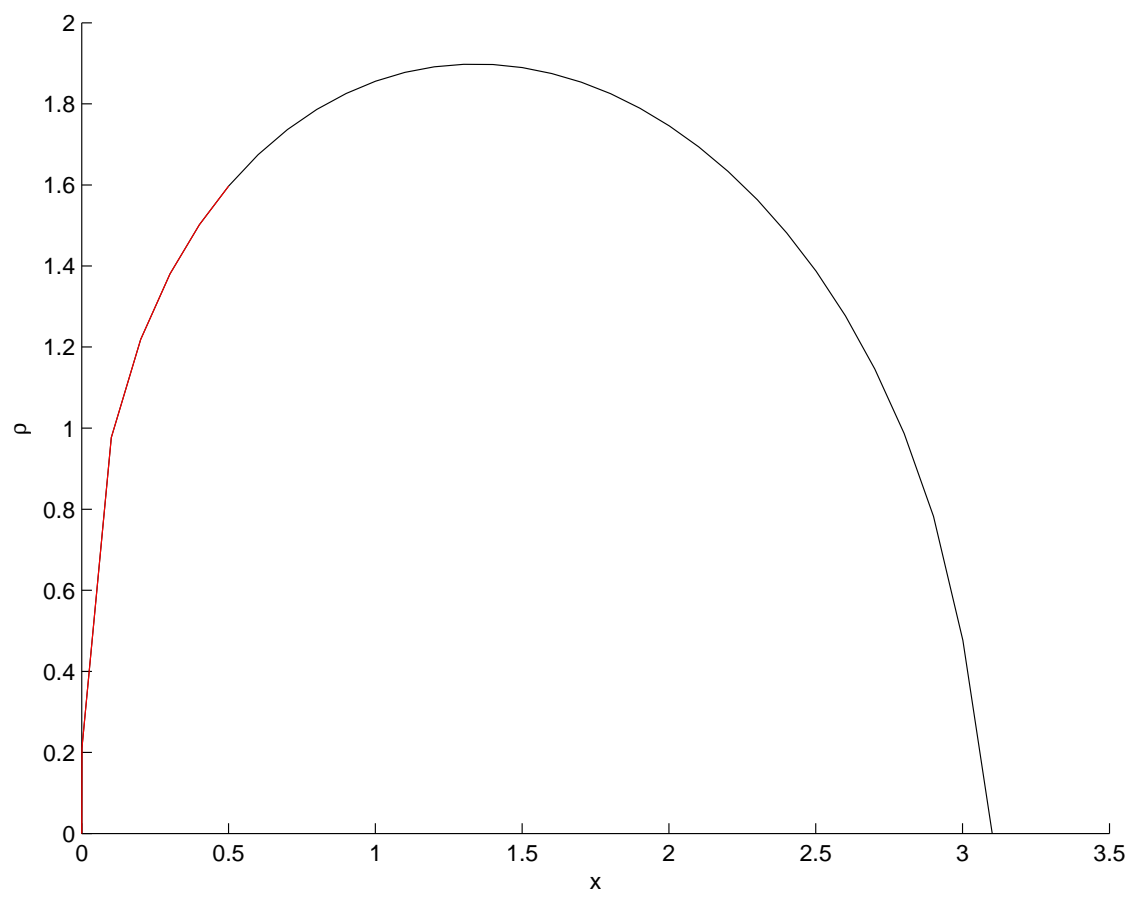

Figure 10. Possible circular orbit solutions as a function of $\mathrm{x}$ from averaged equations. Red $=$ Stable. Black $=$ Unstable.

To test this stability criteria, example simulations of stable and unstable solutions as determined by equation 65 were performed and their trajectories were plotted on the appropriate zero-velocity curve. Figure 11 shows a stable trajectory staying close to the equilibrium point. Note that the zero-velocity curves are computed for the averaged equations with a slightly different energy (a result of terms which have been averaged out) from the non-averaged equations yielding a curve around the averaged equilibrium solution. Figure 12 shows an unstable trajectory wandering far from the equilibrium point as expected and eventually escaping.

\section{Stability in Presence of Outgassing Jet}

Until this point, the analysis presented could be applied to any small body without disturbing forces other than solar radiation pressure. Let's shift out focus to a comet with outgassing jets which produce a varied pressure field in the vicinity of the nucleus. If we consider that the spacecraft passes through an outgassing jet field, we can determine a bound on the strength of the jet for which the spacecraft is contained within 


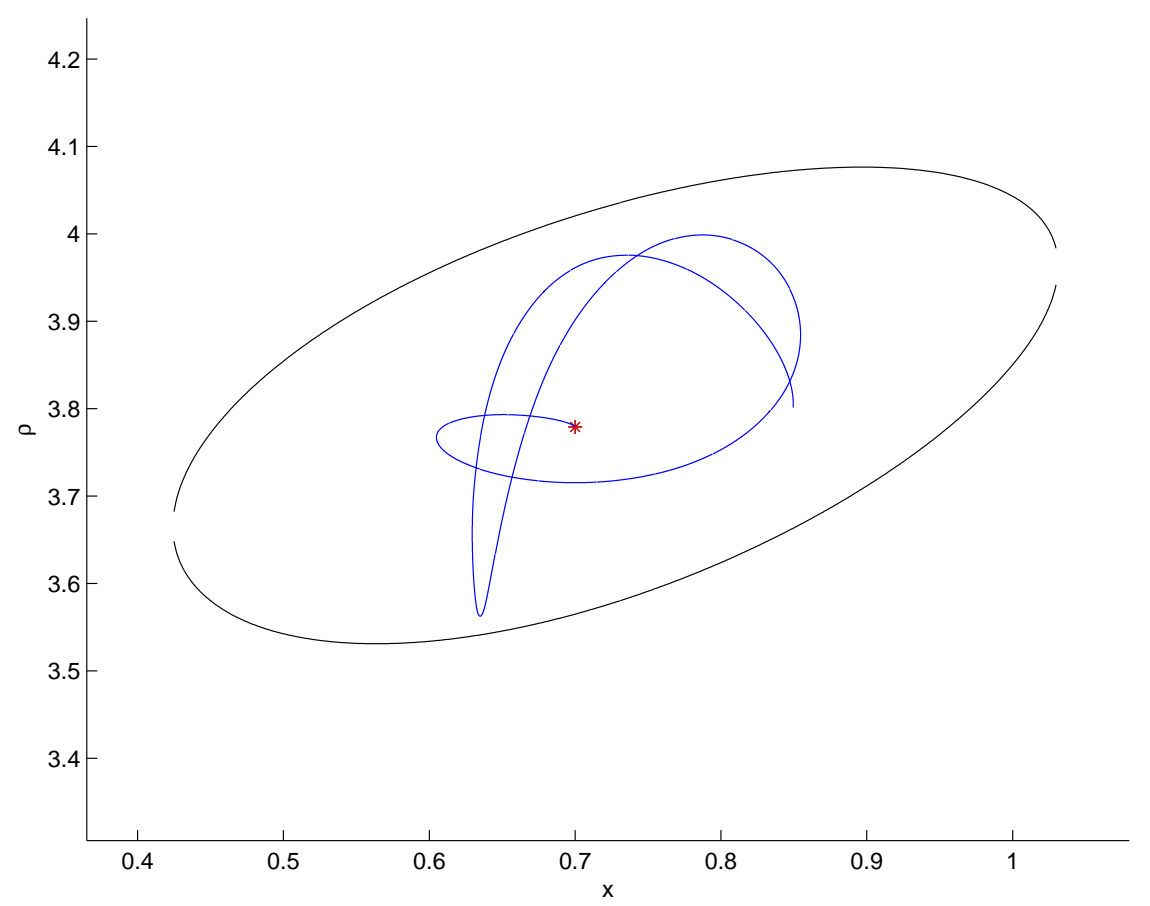

Figure 11. Stable trajectory plotted on zero-velocity curves. Black $=$ trajectory energy. Red $=$ averaged equilibrium solution energy.

an area about a stable equilibrium solutions. The acceleration due to an outgassing jet will be considered as a radial force with the acceleration vector defined as: ${ }^{5}$

$$
\vec{a}_{o j}=\frac{A_{o g}}{|\vec{r}|^{3}} \vec{r}
$$

where $A_{o g}$ is the strength of the outgassing jet. The new equations of motion become:

$$
\ddot{\vec{r}}_{I}=\frac{\partial U}{\partial \vec{r}_{I}}+g \hat{d}+\vec{a}_{o j}
$$

When broken down into cylindrical coordinates, it becomes obvious that only the $x$ and $\rho$ directions are affected by the outgassing jet's acceleration.

$$
\begin{aligned}
\ddot{x} & =2 \omega(\dot{\rho} \cos \theta-\rho \dot{\theta} \sin \theta)+3 \omega^{2} x+g-\frac{x \mu}{r^{3}}+\frac{x A_{o g}}{r^{3}} \\
\ddot{\rho} & =-2 \dot{x} \omega \cos \theta+\rho \dot{\theta}^{2}-\rho \omega^{2} \sin ^{2} \theta-\frac{\rho \mu}{r^{3}}+\frac{\rho A_{o g}}{r^{3}} \\
\rho \ddot{\theta} & =2 \dot{x} \omega \sin \theta-2 \dot{\rho} \dot{\theta}-\rho \omega^{2} \sin \theta \cos \theta
\end{aligned}
$$

Therefore the new Jacobi integral is:

$$
J_{o g}=\frac{1}{2}\left(\dot{x}^{2}+\dot{\rho}^{2}+\rho^{2} \dot{\theta}^{2}\right)-\frac{\mu}{r}-\frac{1}{2} \omega^{2}\left(3 x^{2}-\rho^{2} \sin (\theta)^{2}\right)-g x+\frac{A_{o g}}{r}
$$

Averaging this over $\theta$ as before yields:

$$
\bar{J}_{o g}=\frac{1}{2}\left(\dot{\bar{x}}^{2}+\dot{\bar{\rho}}^{2}\right)-\frac{\mu}{\bar{r}}-\frac{1}{2} \omega^{2}\left(3 \bar{x}^{2}-\frac{1}{2} \bar{\rho}^{2}\right)-g \bar{x}+\frac{h^{2}}{2 \bar{\rho}^{2}}+\frac{A_{o g}}{\bar{r}}
$$

As determined previously, the stable equilibrium solution has a conjugate $h$ valued unstable equilibrium solution which defined the closure point on the zero velocity curve with increased energy. Define $C_{s}$ as the 


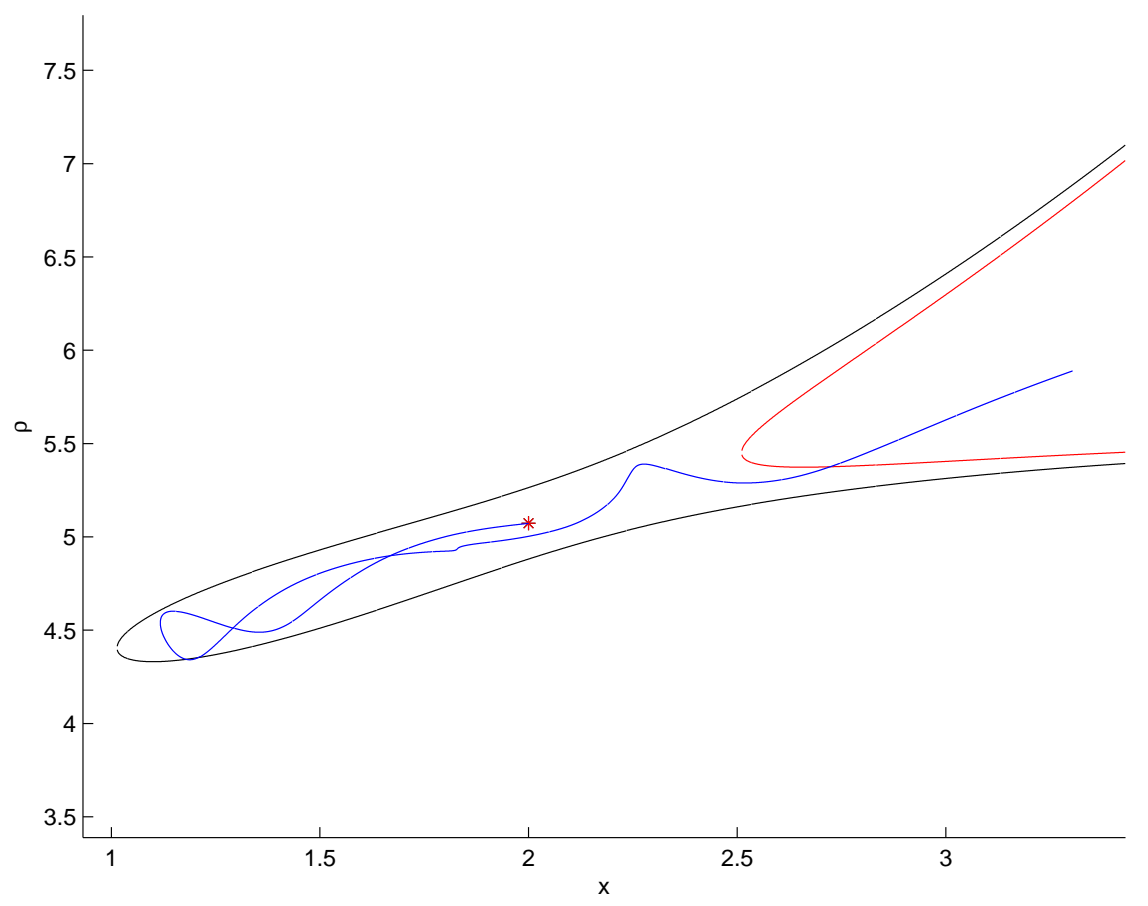

Figure 12. Unstable trajectory plotted on zero-velocity curves. Black $=$ trajectory energy. Red $=$ averaged equilibrium solution energy.

energy associated with a stable equilibrium solution with angular momentum magnitude, $h$, and $C_{u}$ as the energy associated with the conjugate unstable equilibrium solution. Therefore the condition $C_{s} \leq C_{o g} \leq C_{u}$, where $C_{o g}$ is the energy while within an outgassing jet, must hold to keep a spacecraft bounded near the stable equilibrium. This implies a bound on $A_{o g}$ :

$$
\frac{A_{o g}}{\bar{r}} \leq C_{u}-C_{s}
$$

or

$$
A_{o g} \leq\left(C_{u}-C_{s}\right) \bar{r}
$$

Therefore, if the strength of an outgassing jet, $A_{o g}$, can be estimated, this condition gives insight into if the spacecraft will remain bounded in the vicinity of the stable equilibrium. For example, figure 13 illustrates a trajectory of a spacecraft which has passed though an outgassing jet with a magnitude which does not violate the criteria and allows for the spacecraft to remain bounded near the equilibrium solution while the magnitude of the outgassing jet in figure 14 is too large and forces an instability in the orbit allowing it to escape.

\section{Orbital Control}

Since it has been shown that stable orbits exist, we now consider controlling these orbits. Several control schemes are presented to restrict the motion of a orbiting spacecraft using a single dimension or two dimensions to define the boundary of the allowable orbit. Three different control boundaries will be considered when developing a control scheme. The first case is for the motion to be restricted by an angle in the cylindrical coordinate formulation of the equations of motion. This produces a long pie shaped allowable area with the possibility of impact with the nucleus. The second case is for the motion to be restricted by a plane in the cartesian coordinate formulation of the equations of motion to restrict the spacecraft from impacting. Finally, boundaries defined by both an angle and a minimum radius which also restrict impacting are tested. In each case, an acceleration is applied when the spacecraft violates the defined boundary to push the spacecraft back into the allowable motion area. 

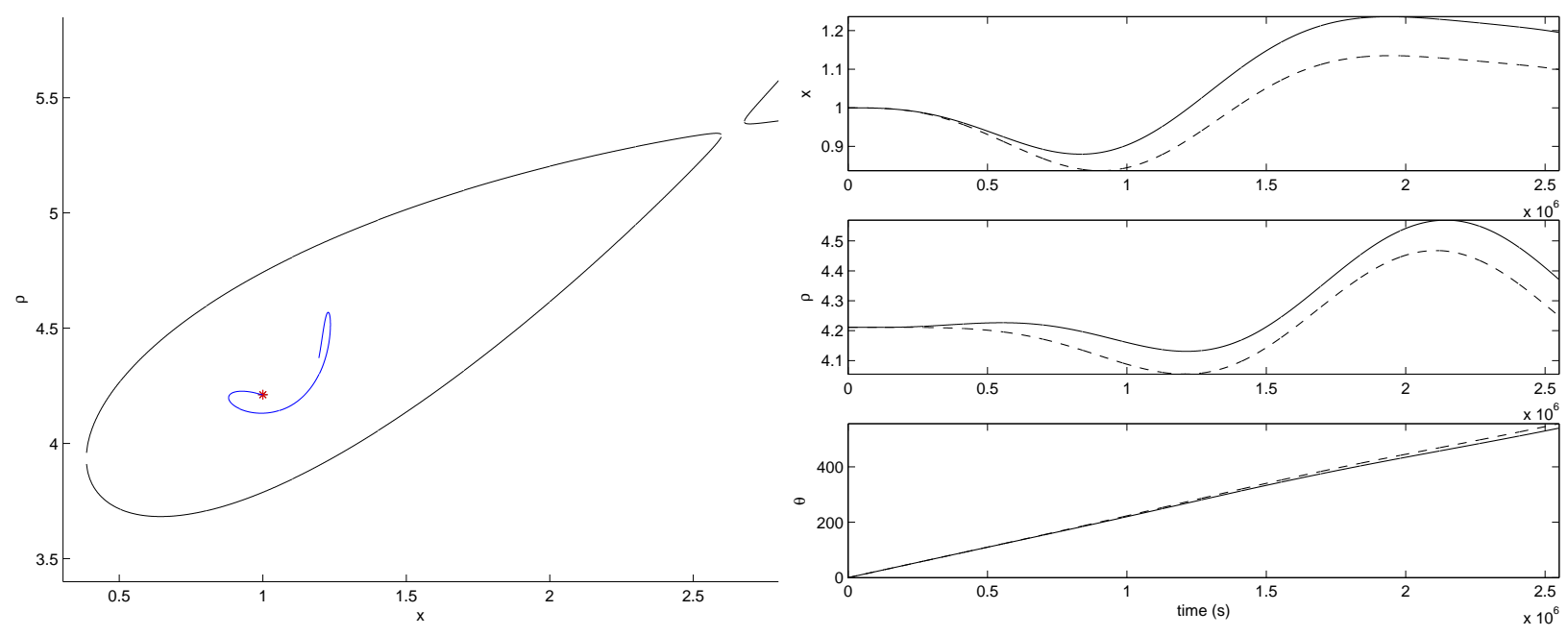

Figure 13. Stable trajectory in the presence of an outgassing jet on appropriate zero velocity curve (two jet passages). Solid = trajectory with outgassing. Dashed = trajectory without outgassing.
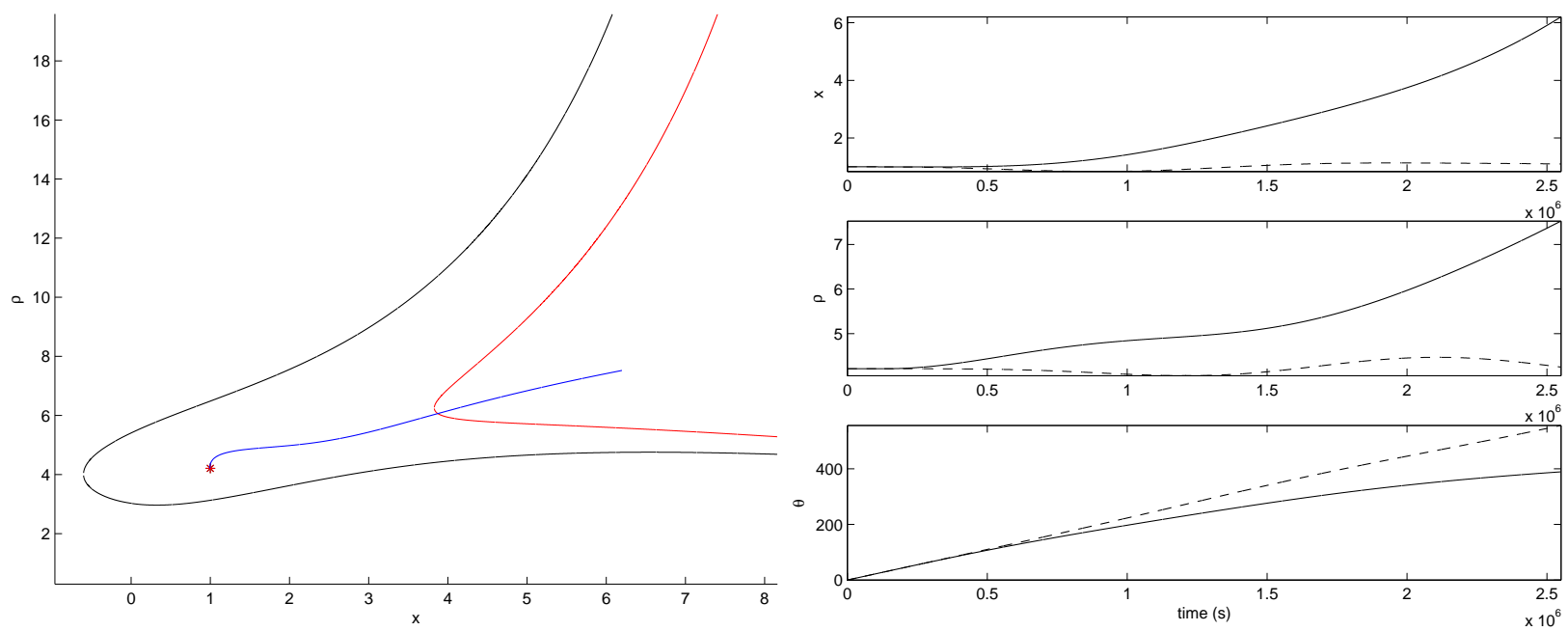

Figure 14. Unstable trajectory in the presence of an outgassing jet on appropriate zero velocity curve (two jet passages). Solid = trajectory with outgassing. Dashed $=$ trajectory without outgassing. 
For our initial analysis we consider a theoretical control method that is commonly used to test the feasibility of the control boundaries, an impulsive thrust. First consider the case where a $\Delta v$ is applied to reverse the direction of the full velocity vector while holding the magnitude constant. This result, as seen in figures 15 and 16, does not maintain the orbit perpendicular to the Sun line although the impulsive is successful at not violating the boundaries.
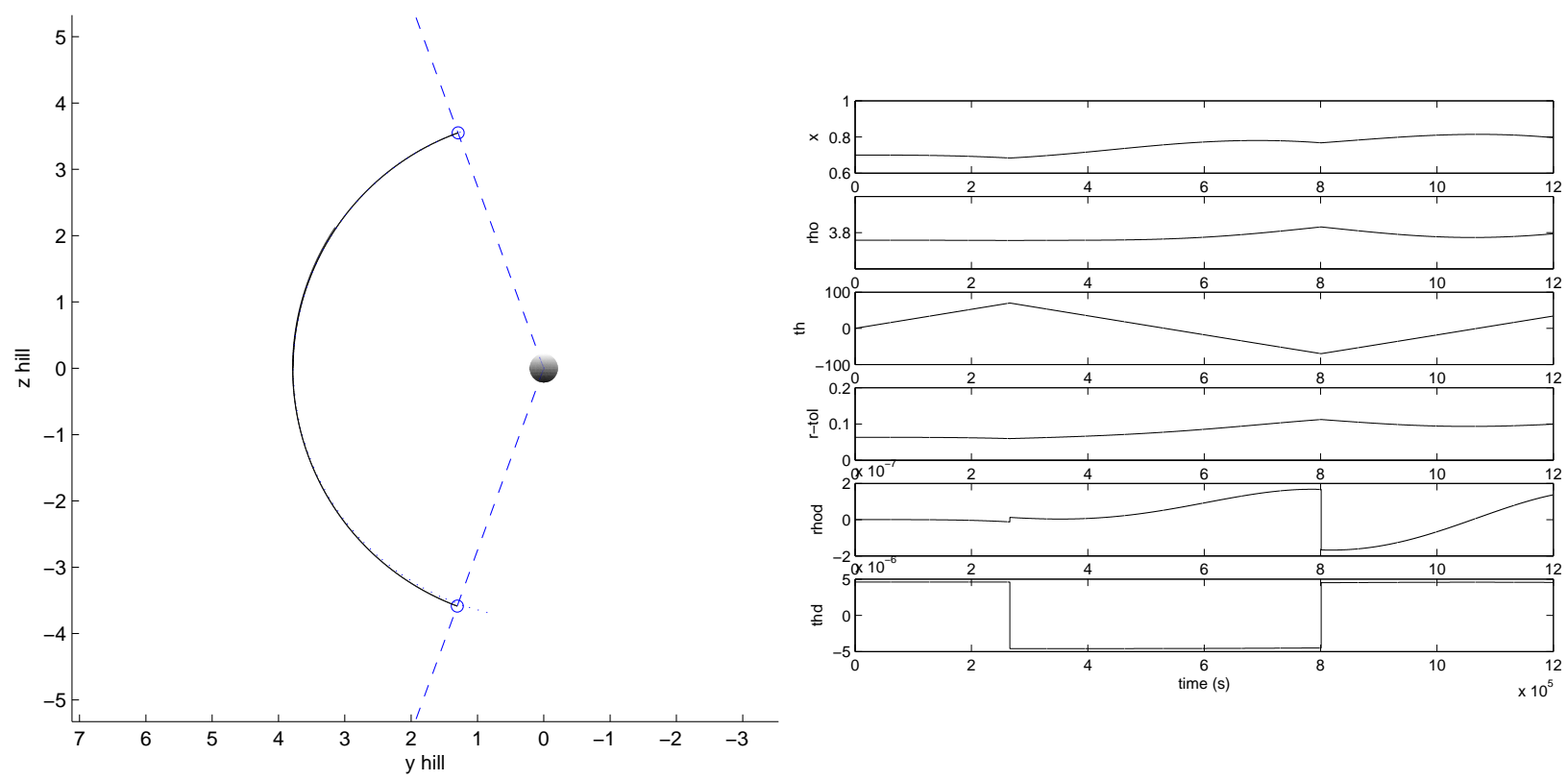

Figure 15. Orbit control with reversed $\theta$ and $\rho$ velocity impulse with angle boundary. Solid $=$ controlled trajectory. Blue = angle boundary. o = impulse location. Position and velocity components of the controlled orbit are also plotted for reference.
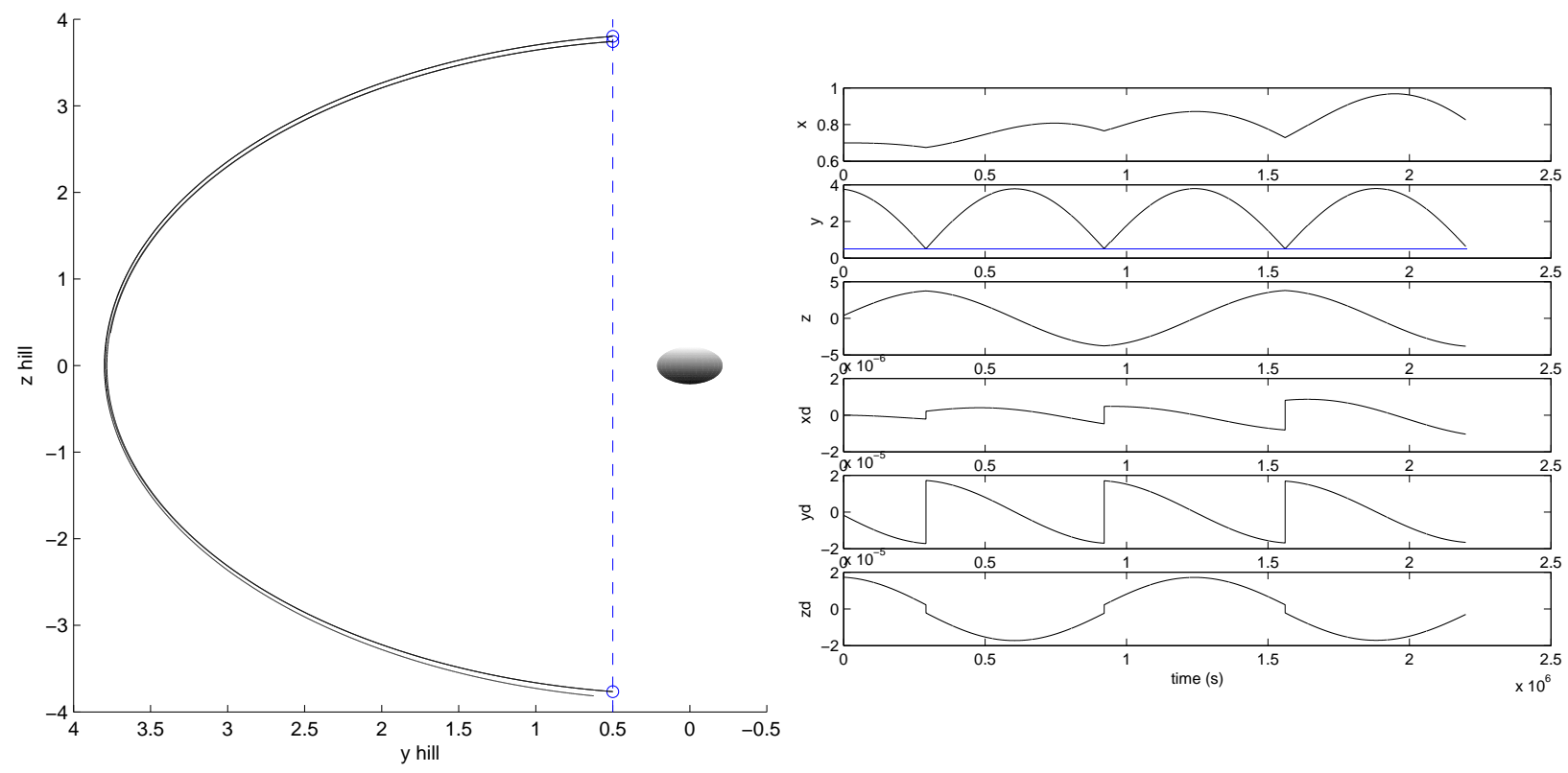

Figure 16. Orbit control with reversed $\theta$ velocity impulse with plane boundary at $y=0.5 \mathrm{~km}$. Solid $=$ controlled trajectory. Blue = angle boundary. o = impulse location. Position and velocity components of the controlled orbit are also plotted for reference with blue defining a component's boundary.

Since the reversal of the full velocity vector performs poorly at maintaining the orbit's attitude, let's consider the exact same impulse maneuver except only reverse the $\dot{y}$ and $\dot{z}$ components (or $\dot{\rho}$ and $\dot{\theta}$ compo- 
nents). This result, as seen in figure 17, also does not maintain the orbit perpendicular to the Sun line but has less deviation than the full velocity reversed maneuver.
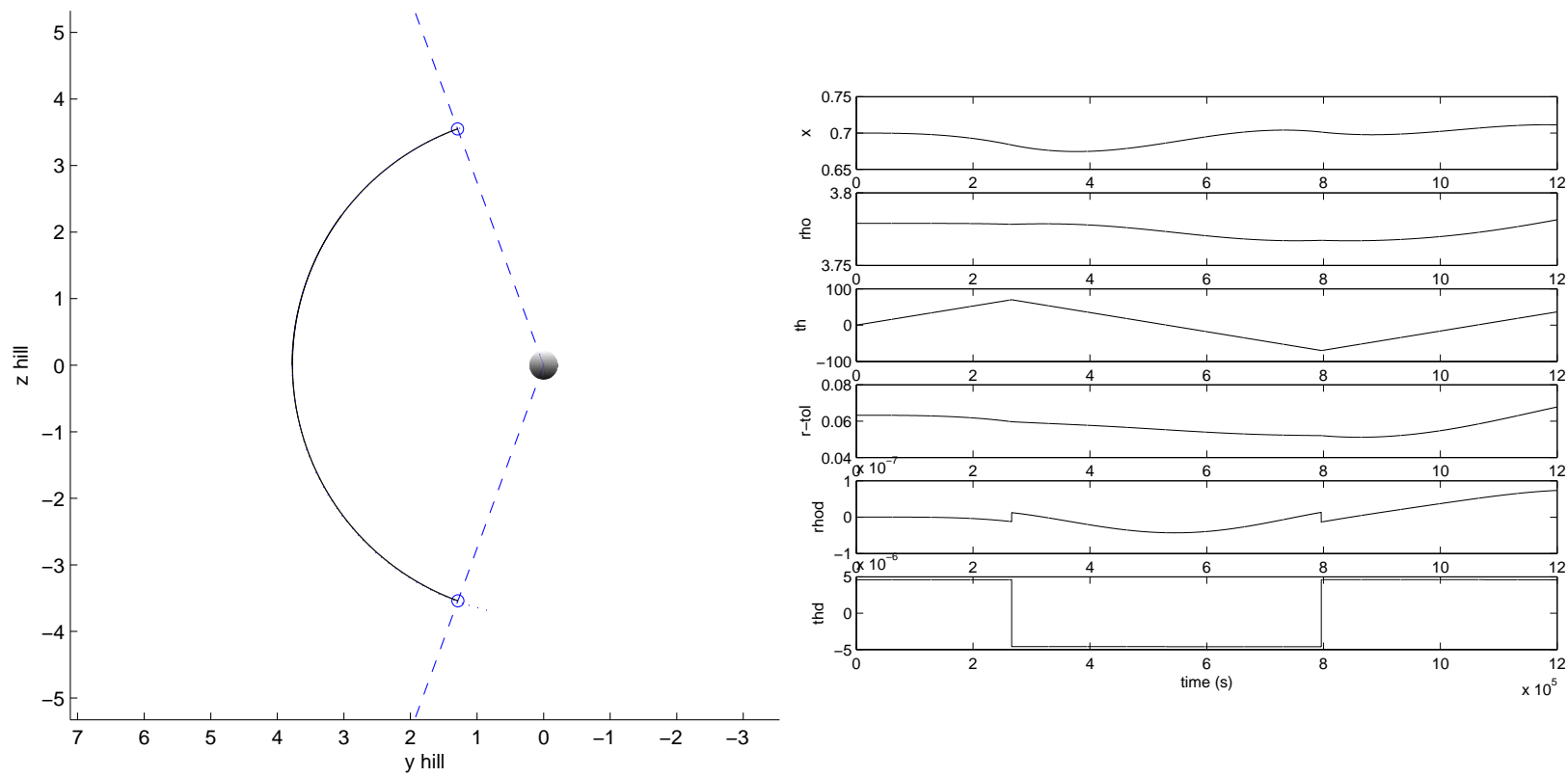

Figure 17. Orbit control with reversed velocity impulse in the $y$ and $z$ directions with angle boundary. Solid $=$ controlled trajectory. Blue $=$ angle boundary. $\mathrm{o}=$ impulse location. Position and velocity components of the controlled orbit are also plotted for reference.

Finally let's consider once again the exact same impulse maneuver except only reverse the $\dot{\theta}$ component:

$$
\Delta v=v_{2}-v_{1}=\rho_{2} \dot{\theta}_{2}-\rho_{1} \dot{\theta}_{1}
$$

where $\rho_{2}=\rho_{1}=\rho$ and $\dot{\theta}_{2}=-\dot{\theta}_{1}=\dot{\theta}$ resulting in:

$$
\Delta v=2 \rho \dot{\theta}
$$

This would ideally result in a final velocity of $-\rho \dot{\theta}$. This control impulse, as seen in figure 18, maintains the $x$ offset similar to the $\dot{\rho}$ and $\dot{\theta}$ reversal but does not maintain the $\rho$ component as well.

Figure 19 shows the comparison of the three impulsive schemes for the angle boundary. It is clear that the reversal of the $\dot{\rho}$ and $\dot{\theta}$ velocity components is the best of the three at maintaining the orbit across all three position components.

The drawback to the boundary defined by angles is that the spacecraft can be contained within the bounded area and still impact the comet. Let's consider now an orbit bounded by the angles as before and also a minimum radius from the body. When the spacecraft encounters the angle boundary, a reversal of the $\dot{\theta}$ velocity component impulse will be applied, while a a reversal of the $\dot{\rho}$ velocity component impulse will be applied when the radius boundary is violated. Figure 20 illustrates the result of this control scheme and figure 21 illustrates the same control scheme with the addition of a reversal of the $\dot{x}$ velocity component also when the minimum radius boundary is encountered. Note that both methods give similar results for the $x$ offset but the method with the additional $\dot{x}$ velocity control maintains the $\rho$ component better.

The control schemes up until this point have been impulsive thrust maneuvers with the main objective of confining the spacecraft within a region. These methods were necessary to illustrate that the spacecraft could be controlled to remain within an allowable region and not escape. Future work includes designing a finite burn control scheme based on the performance results of these impulsive maneuvers.

\section{Conclusions}

We have shown that on average Sun synchronous circular orbits exist in the Hill equations of motion with solar radiation pressure. An stability analysis was performed and criteria was given to produce stable

$$
16 \text { of } 19
$$



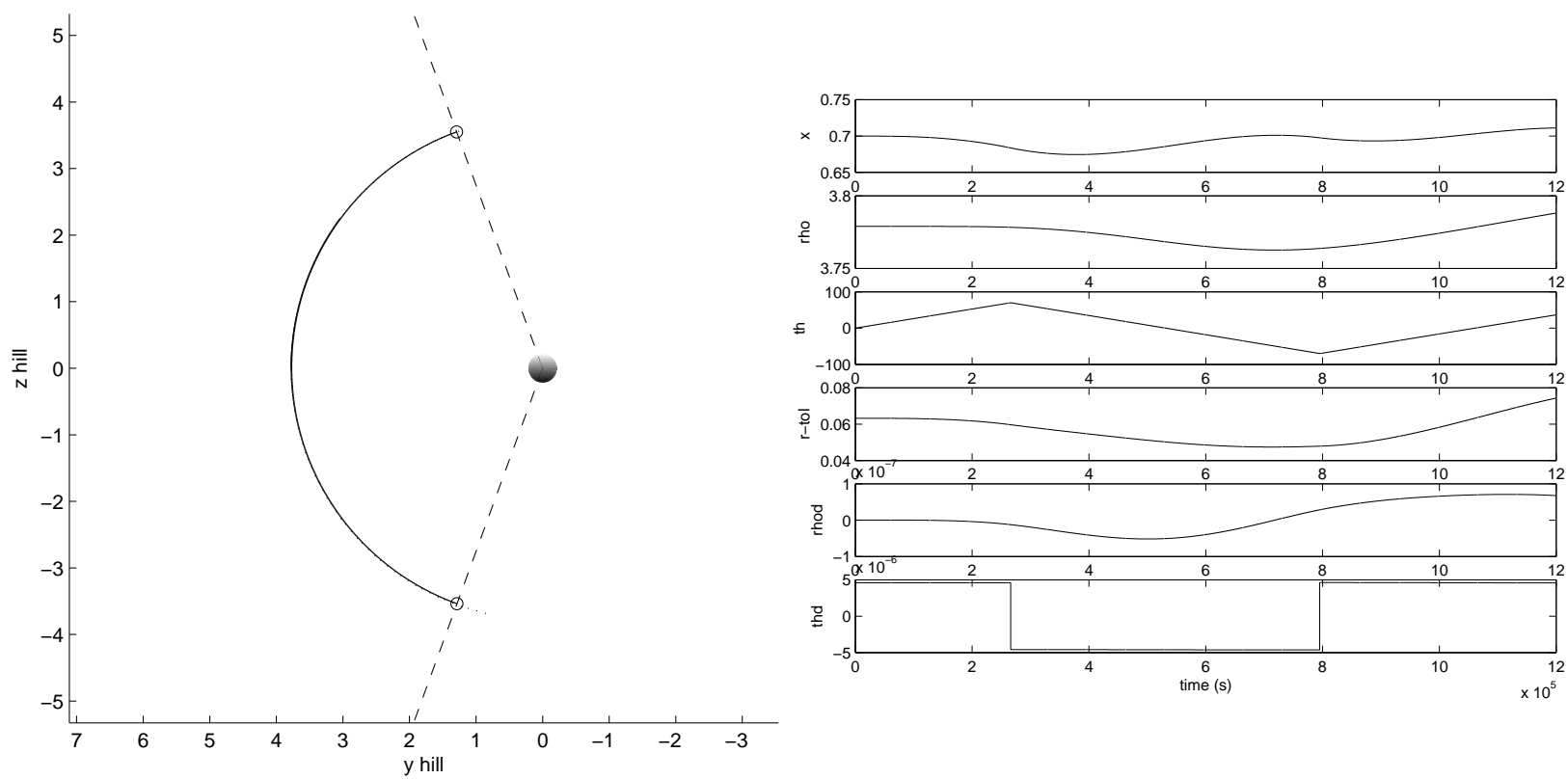

Figure 18. Orbit control with reversed velocity impulse in the $y$ and $z$ directions with angle boundary. Solid $=$ controlled trajectory. Blue $=$ angle boundary. $\mathrm{o}=$ impulse location.

orbits for a given system. The calculations of the zero velocity curves also gave insight into the stability of these orbits as well as under the pressure from an outgassing jet. Multiple control schemes were presented to restrict the motion of the spacecraft within a defined bounded area.

\section{Acknowledgements}

We acknowledge support for this research from the JPL MMR program.

\section{References}

${ }^{1}$ Announcement of Opportunity: New Frontiers Program 2003 and Missions of Opportunity (AO-03-OSS-03), NASA, October 2003, http://research.hq.nasa.gov/code_s/nra/current/A0-03-0SS-03/index.html] [retrieved November 2007].

${ }^{2}$ Dankowicz, H., "Some Special Orbits in the Two-Body Problem with Radiation Pressure," Celestial Mechanics and Dynamical Astronomy, Vol. 58, 1994, pp. 353-370.

${ }^{3}$ Scheeres, D. J. and Marzani, F., "Spacecraft Dynamics in the Vicinity of a Comet," Journal of the Astronautical Sciences, Vol. 50, No. 1, 2002, pp. 35-52.

${ }^{4}$ Scheeres, D. J., "Satellite Dynamics about Small Bodies: Averaged Solar Radiation Pressure Effects," Journal of the Astronautical Sciences, Vol. 47, No. 1, 1999, pp. 25-46.

${ }^{5}$ Byram, S. M. and Scheeres, D. J., "Models for the Comet Dynamical Environment," Journal of Guidance, Control, and Dynamics, Vol. 30, No. 5, 2007, pp. 1445-1454.

${ }^{6}$ Scheeres, D. J., "Dynamics about Uniformly Rotating Triaxial Ellipsoids: Applications to Asteroids," ICARUS, Vol. 110, 1994, pp. 225-238.

${ }^{7}$ Villac, B. F. and Scheeres, D. J., "Escape Trajectories in the Hill Three-Body Problem and Applications," Journal of Guidance, Control, and Dynamics, Vol. 26, No. 2, 2003, pp. 224-232. 

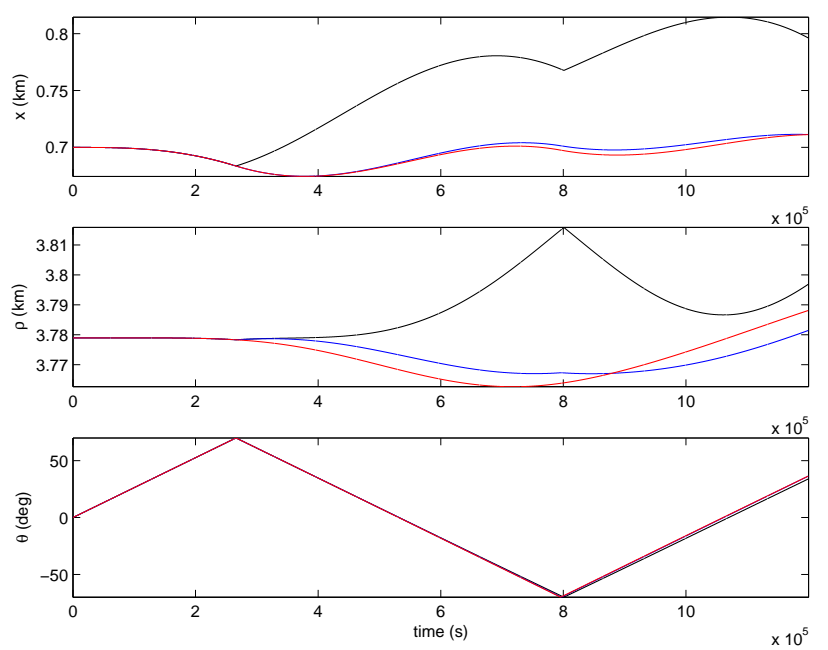

Figure 19. Orbit control comparison with angle boundary. Black $=$ full velocity reversal. Blue $=\dot{\rho}$ and $\dot{\theta}$ reversal. Red $=\dot{\theta}$ reversal.
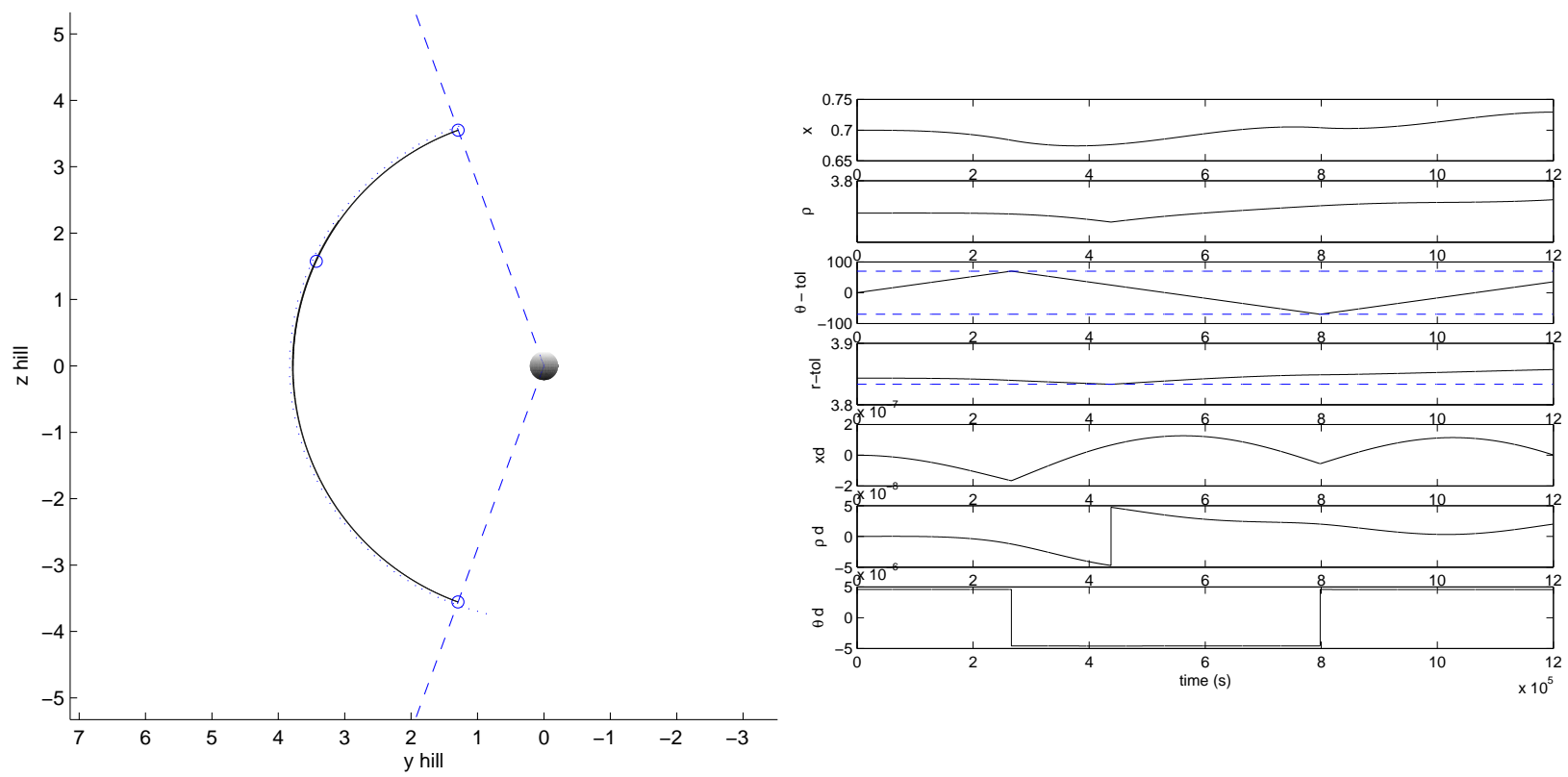

Figure 20. Orbit control with reversed velocity impulse in the $\dot{\theta}$ direction with the angle boundary and $\dot{\rho}$ direction with minimum radius boundary. Solid $=$ controlled trajectory. Blue $=$ angle boundary. $0=$ impulse location. 

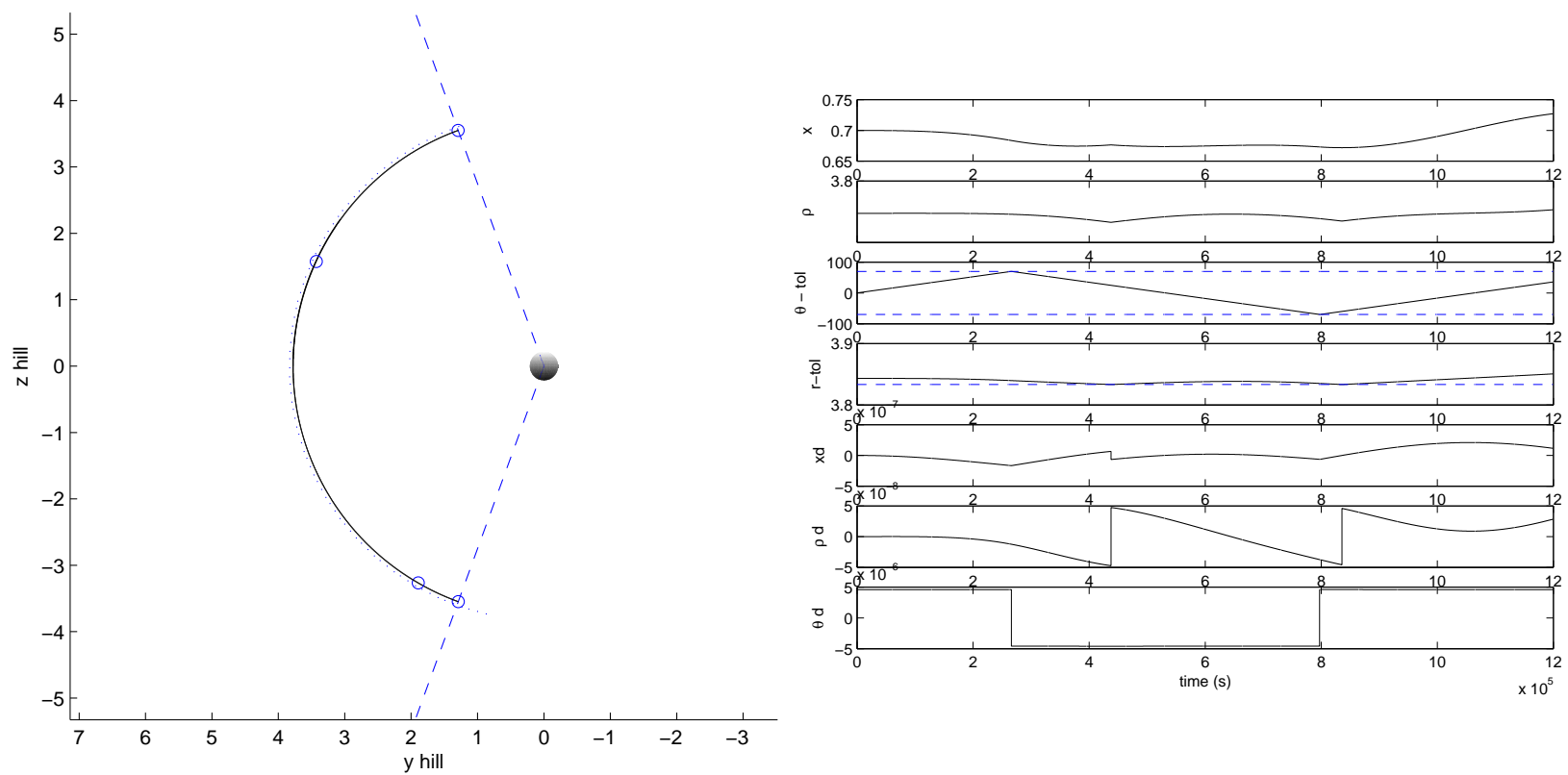

Figure 21. Orbit control with reversed velocity impulse in the $\dot{\theta}$ direction with the angle boundary and $\dot{\rho}$ direction with minimum radius boundary. Solid $=$ controlled trajectory. Blue $=$ angle boundary. $o=$ impulse location. 